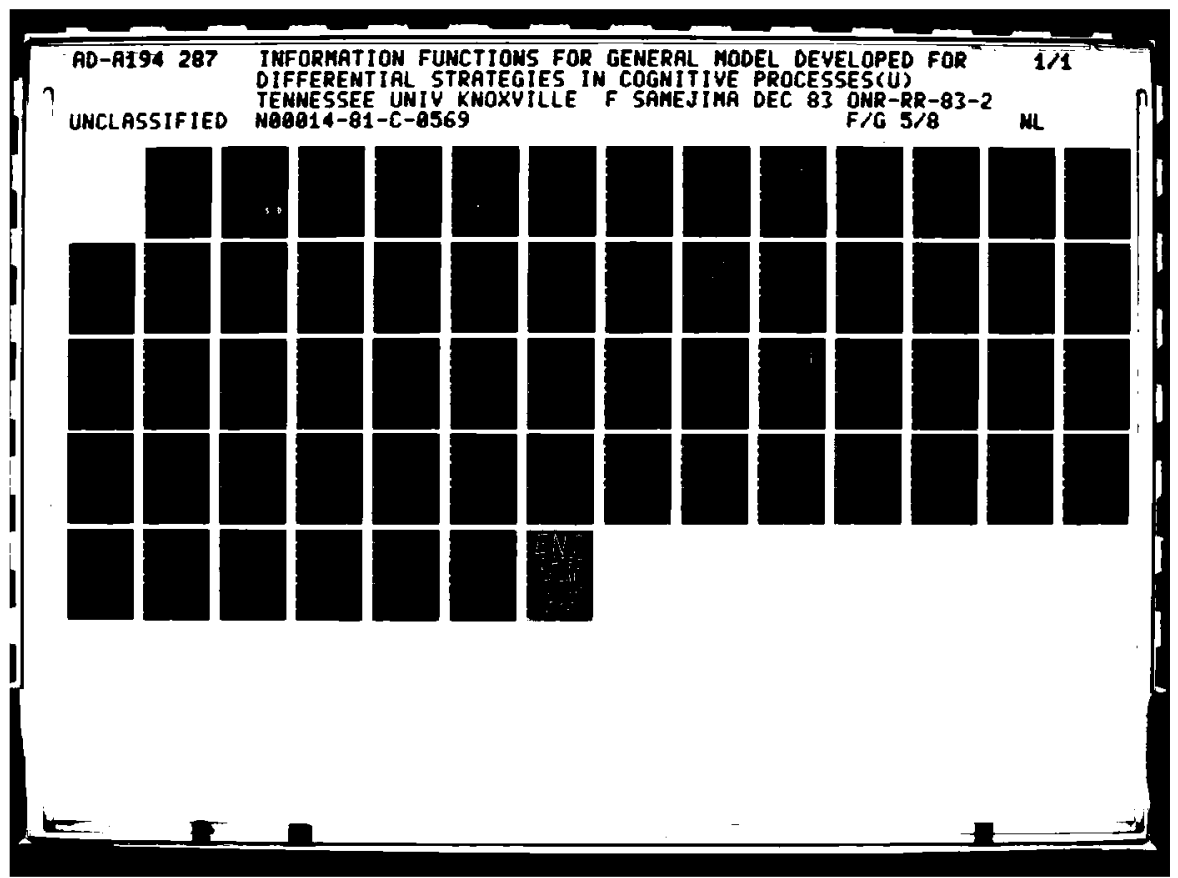




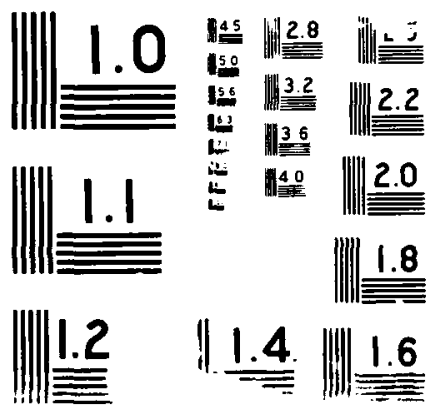




\section{AD-A 194287}

$$
\text { bith in }
$$

\section{INFORMATION FUNCTIONS FOR GENERAL MODEL DEVELOPED FOR DIFFERENTIAL STRATEGIES IN COGNITIVE PROCESSES}

FUMIKO SAMEJIMA

\section{UNIVERSITY OF TENNESSEE}

KNOXVILLE, TENN. 37996-0900

DECEMBER, 1983

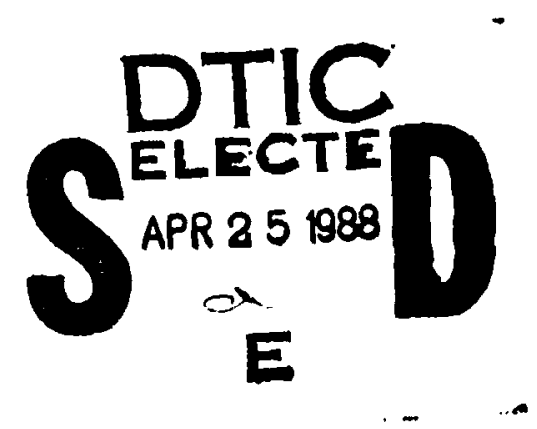

Prepared under the contract number N00014-81-C-0569, NR 150-467 with the

Personnel and Training Research Programs

Psychological Sciences Division

Office of Naval Research

Approved for public release; distribution unlimited.

Reproduction in whole or in part is permitted for

any purpose of the United States Government. 


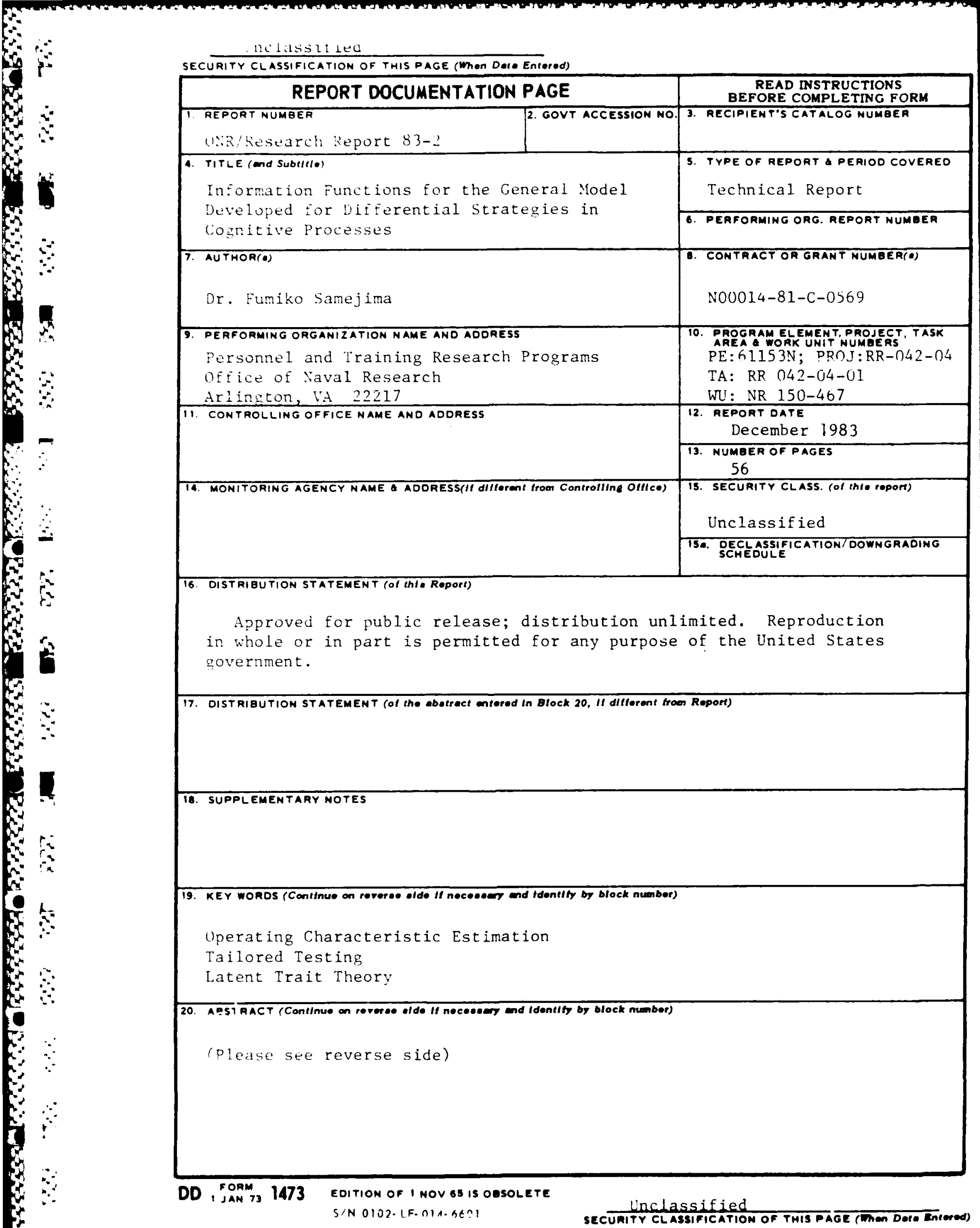


In the preceding research report, ONR/RR-83-2, entitled "A Latent Trait Model for Differential Strategies in Cognitive Processes," a general model was proposed in the context of latent trait theory which deals with differential strategies in cognitive processes. With that general model, it is necessary to define the iliformation functions expanded from those defined for the models on the graded response level. In the present report, such an attempt is made and described. 
TABLE OF CONTENTS

Page

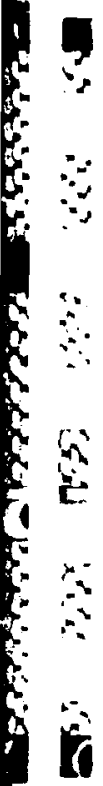

I Introduction

1

II General Model for Differential

5

Strategies

II Homogeneous Case

8

IV Information Provided by Differential

12

$\checkmark$ Discussion

50

References

51
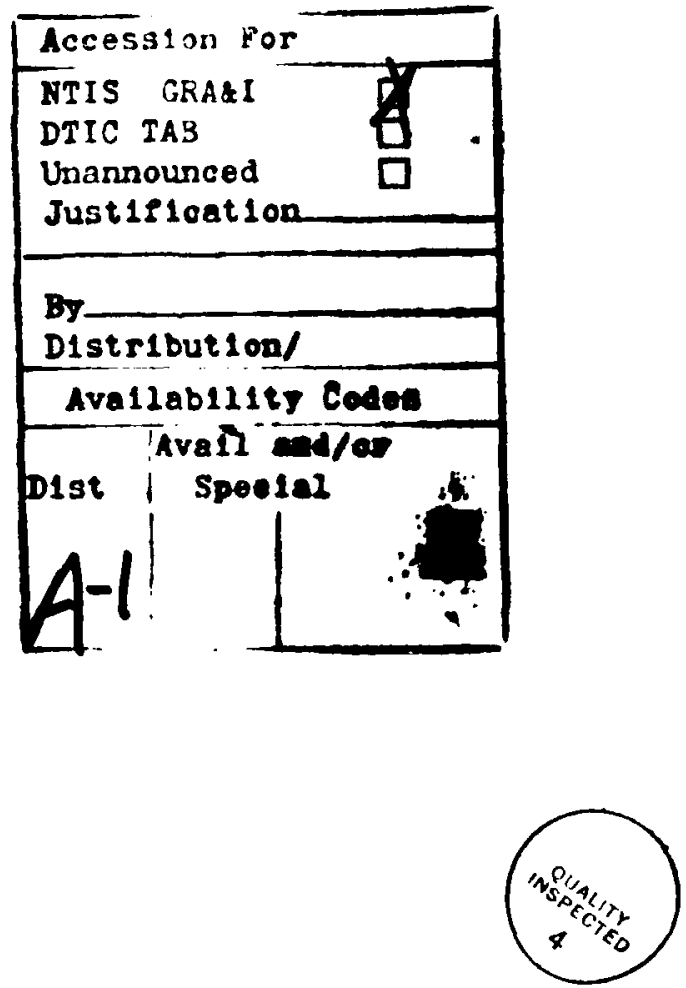


\section{Introduction}

One of the main objectives of this research project is to "bridge" psychometrics with cognitive psychology through the advancement of latent trait theory. With the rapid progress of microcomputers in the past decade and accompanied decreases in their cost, many scientific investigations which were considered practically impossible in the past are now within our reach. Thus in many areas of cognitive psychology, where researchers used to conduct their research using relatively small samples of subjects, we can plan our research on a much larger scale. Time is coming, therefore. that latent trait theory will find its way to contribute to the progress of cognitive psychology.

Some cognitive psychologists, who have tried to approach psychometric theories, say that they do not provide them with theories and methods with which they can deal with differential strategies. They are not exactly right, however. As early as in the late nineteen-sixties, the heterogeneous case of the graded response level in the context of latent trait theory was proposed (Samejima, 1967) as a model for cognitive processes. Some useful hints for differential strategies are also seen (Samejima, 1972, Section 3.4) under the title "Multi-correct and multiincorrect responses."

In the preceding research report (Samejima, 1983), a latent trait model for differential strategies in cognitive processes was proposed. In so doing, digraphs were used to represent cognitive processes, with traversing each path indicating the completion of each successful strategy.

Let $\theta$ be a unidimensional latent trait, or "ability", which assumes 
any real number. so that we can write

Suppose there is only one successful strategy for solving the problem $g$, and that we need $m_{g}$ sequential subprocesses. Let $y_{g}$ denote the attainment category or attainment score. One must successfully follow air the $\mathrm{mg}$ sequential subprocesses in order to solve the problem $g$, so the attainment category $y_{g}$ assumes integers, 0 through $\left(m_{g}+1\right)$, with $y_{g}=0$ indicating that the individual subject has successfully followed none of the subprocesses, and with $y_{g}=m_{g}$ meaning that he has completed all $\mathrm{mg}$ subprocesses required to solve the problem. The additional attainment score, $\left(\mathrm{m}_{\mathrm{g}}+1\right)$, indicates that the subject has successfully followed the additional subprocess which does not exist but is hypothesized at the end of the entire sequence of subprocesses. Since no one can accomplish this, the conditional probability, given $\theta$, with which the subject obtained the attainment score $y_{g}$ equals zero, regardless of a given value of $\theta$. With this setting, we can see that the general graded response model can readily be applied to the single strategy case of problem solving. It is a fairly common phenomenon, however, that there exist more than one way of solving a problem. Our main objective is to approach a general model for the multiple strategy case, or differential strategies, in the context of latent trait theory.

Let $w$ denote the number of successful strategies for solving the problem $g$, and $y_{g i}\left(=0,1, \ldots, m_{g i}\right)$ be the attainment score for the 
subject who has taken the strategy $i(=1,2, \ldots, w)$ for solving the problem g. Figure 1-1 presents an example of the digraph representing five differential strategies, i.e., $w=5$, which was used as one of the examples in the previous research (Samejima, 1983). In this figure, for the purpose of illustration, the set of attainment scores for the strategies No. 1 and 4 are attached to the edges.

In our cognitive process we often falter or choose wrong strategies which do not lead to the solution of the problem at all. Even if the subject took a wrong strategy, however, he may become aware of his mistake and come back to a previous point in the path and try another strategy. There are a great many other varieties of paths, trails and walks, each of which might represent a specified subject's cognitive process. It is obvious, however, that traversing on cycles or taking those unsuccessful paths, trails and walks will not improve the subject's degree of attainment in solving the problem; they should be more or less ignored. It is a kind of directed graph which contains several paths representing different strategies, joining a common initial endpoint with the distinct other endpoints. Since no one can surpass a solution point, it also represents a hypothesized attainment score which no one can obtain.

The differential strategy tree was proposed to represent both successful and unsuccessful strategies for solving a specified problem. Each tree starts from a single "nothing point" and, if we ignore all unsuccessful strategies, ends with as many "solution points" as we have different successful strategies.

Figure 1-2 presents the differential strategy tree for the example 


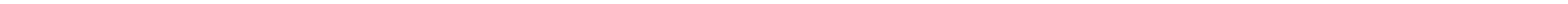


given in Figure 1-1. in which only successful strategies are drawn, and the attainment is score assigned to each edge. Thus in our example, we have five successful strategies and five solution points.

\section{ii. General Model for Differential Strategies}

A general model for differential strategies concerns the assignment if ar operating characteristic to each attainment score $y_{g i}$ of each of the $w$ strategies for solving the problem $g$. By such an operating characteristic we mean the conditional probability with which the subject of trait $\theta$ chooses the strategy $i$ and obtains the attainment score $\because a j$. We notice, however, that in general, if the subject's performance stopped before branching, there is no way to decide which of the two or more strategies he would have taken. For example, $\left(\begin{array}{lll}s_{1} & s_{2}\end{array}\right)$ and $\left(t_{1} t_{2}\right)$ in Figure 1-2 are a single edge, and so are $\left(v_{1} v_{2}\right)$ and $\left(w_{1} w_{2}\right)$. Thus .. must azsign a single operating characteristic for each edge of the differential strategy tree. Since each edge represents a union of one or mice attainment scores, the operating characteristic is to be assigned to each union. For instance, following an appropriate model, a single ooerating characteristic will be assigned to the union of $\mathrm{y}_{\mathrm{gi}}=0$ for $i=1,2.3,4,5$, and the same model will provide us with an operating characteristic solely for $y_{g i}=3$. For convenience, we shall choose the smallest $i$ in each union, and let $y_{\text {gis }}^{\star}$ denote such a union with $s$ for tne actual attainment score.

Let $P_{y_{g}^{\star} i}^{\star}(\cdot)$ be the conditional probability assigned to the union of attainment scores $y_{g s i}^{\star}$, with which the subject of trait $\theta$ chooses a strategy which belongs to $\mathrm{y}_{\mathrm{gs}}^{*} \mathrm{i}$ and obtains the attainment score $\mathrm{s}$ or 
greater. We shall call this function the cumulative operating characteristic of the attainment score union $\mathrm{y}_{\mathrm{gsi}}^{*}$. By virtue of the natures of the attainment scores 0 and $\left(m_{g i}+1\right)$, we have

$$
p_{y_{g s i}^{*}(\theta)} \begin{cases}=1 & s=0 \\ =0 & s=m_{g i}+1\end{cases}
$$

for the entire range of $\theta$.

The operating characteristic, $P_{y^{\star} s i}(\theta)$, defined for the union of the attainment scores $y_{g s i}^{\star}$ is given by

$$
P_{y_{g s i}^{\star}}(\theta)=P_{y_{g s i}^{*}}^{*}(\theta)-\sum_{j^{\star}} P_{y_{g}^{\star}(s+1) j}^{*}(\theta)
$$

where $\sum_{j *}$ indicates the summation over all the strategies $j$ branching from the point which lies immediately after the line representing $y_{g s i}^{*}$ in the differential strategy tree. This operating characteristic can be considered as the likelihood function in estimating the subject's latent trait $\theta$.

When there are more than one problem to solve, i.e., $g=1,2, \ldots, n$, the maximum likelihood estimation of the subject's latent trait can be performed on the basis of the response pattern $V$, such that

$$
V^{\prime}=\left(y_{1 i_{1}}, y_{2 i_{2}}, \ldots, y_{g i_{g}}, \ldots, y_{n i_{n}}\right)
$$

for the $n$ problem solving tasks, where $i_{g}$ is a strategy for solving the problem $g$ and $y_{g i}$ is the attainment score when the subject chooses the strategy ig for solving the problem $g$, provided that the conditional 
$\therefore$

$\therefore$

independence of the distributions of the attainment scores across the different tasks, given: holds. Let $P_{V}(\theta)$ be the operating characteristic of the specific response pattern $V$. We can write

$(2 .+4)$ $P_{v i}(: *)=\|_{*} P_{y_{g S i}^{*}}(: i)$

$\because$

where $\quad$ * incicates the multiplication over every union $y_{\text {gsi }}^{\star}$ to which an element of $V$ belongs.

It is beneficial to search for a family of models which provide us with a unique maximum for every possible response pattern given by (2.3). Tnis can be done as a generalization of the unique maximum condition proposed for the graded response model (cf. Samejima, 1969, 1972).

The basic function. $A_{y_{g S i}^{*}}(\cdot)$, for the union of attainment scores $y_{G j i}^{\star}$ is defined by

$(2 . j)$

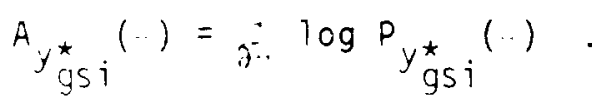

The maximum likelihood estimate, $\therefore$, of the subject's latent trait based upon his response pattern is given as the solution of the likelihood equation such that

$$
\begin{aligned}
& \therefore \log P_{V}()=\because \ldots \log P_{y}=\ldots(\cdot) \\
& =A_{y^{\star}} \underset{Z_{S i}}{ }(1),
\end{aligned}
$$

$\because$

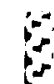

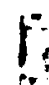


where $\Sigma$ indicates the summation over every union $\mathrm{y}_{\mathrm{gs}}^{\star} \mathrm{i}$ to which an element of $V$ belongs. A sufficient condition that a unique modal point exists for the likelihood function $P_{V}()$ of each and every response pattern $V$ is that this basic function is strictly decreasing in $a$ with non-negative and non-positive values as its two asymptotes, respectively. for every union $y_{0 s i}^{\star}$. This can be shown in the same way that we did for the basic function $A_{x_{1}}\left(\right.$ ) of the graded item score $x_{g}$ (cf. Samejima, 1969). For brevity. Sometimes we call this condition the unique maximum condition.

Similarities between the differential strategies in problem solving and the multi-correct responses in testing are obvious. If we consider two or more different strategies which lead to the solution of the problem as two or more different answers to a question, then they will be treated as multi-correct responses. We can see that the concept of multi-correct responses can be transferred to differential strategies, when there exist more than one successful strategy in solving the problem.

\section{Homogeneous Case}

The Homogeneous case of the graded response level has been deveioped and discussed (Samejima, 1972) as a generalization of a family of models on the dichotomous response level. Sufficient conditions that a model provides us with a unique modal point for the likelihood function of each and every response pattern have been investigated. In the homogeneous case, a sufficient condition is that, for an arbitrary item score $x_{y}(\neq 0)$. the cumulative operating characteristic $P_{x_{\theta}}^{*}(\theta)$ is strictly increasing in $\theta$ with zero and unity as its two asymptotes, i.e., of Type A, and its 
asymptotic basic function, $\hat{A}_{x}(\theta)$, which is defined by

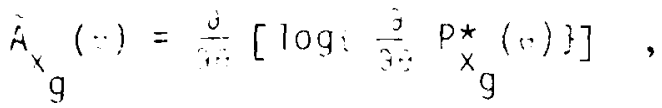

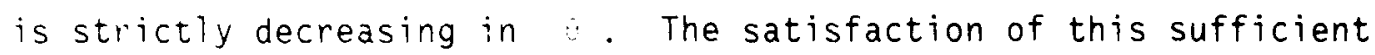
condition also implies two desirable features of the model such that:

1) the operating characteristic of each graded item score of each item has a single modal point, and 2) those modal points for a single item are arranged in the same order as the item score itself. The normal ogive and logistic models, which have been generalized from the corresponding models on the dichotomous response level, are two examples of the models which satisfy the above sufficient condition.

These models of the homogeneous case on the graded response level can be generalized to provide us with those which belong to the general model of differential strategies. Let $\Psi(\theta)$ be a function of Type A. We shall consider the cumulative operating characteristic, $\mathrm{P}_{\mathrm{gsi}^{*}}^{*}(\theta)$, of the union of attainment categories $y_{g s i}^{*}$ such that

$$
P_{y_{g S i}^{\star}}^{*}(\theta)=\beta_{y_{g s i}^{\star}} \psi\left(\theta-\alpha_{y}^{\star}\right),
$$

where

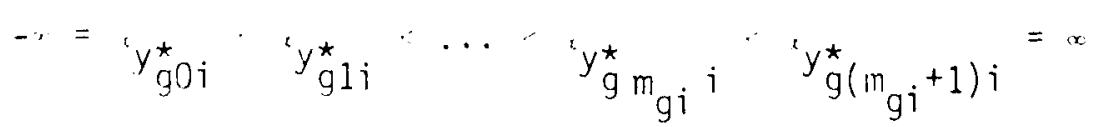

for every strategy, and $\tilde{\sim}_{g s i}^{\star} \underset{g s}{ }$ is a constant which satisfies 


$$
\begin{cases}{ }_{y_{g s i}^{\star}=1} & s=0 \\ \sum_{j \star} z_{y_{g S j}^{\star}={ }^{3} y_{g(s-1) i}^{*}} & s=1,2, \ldots, m_{g i},\end{cases}
$$

with $\sum_{j *}$ indicating the summation over all the strategies $j$ branching from the point of the differential strategy tree which is located right after the edge representing the union $y_{g}^{*}(s-1) i$ in the differential strategy tree. From (3.4) it is obvious that, as far as there is no branching, ${ }^{z} y_{g s i}^{*}=B_{y}^{*}(s-1) i$.

A sufficient condition that the model satisfies the unique maximum condition is: 1) that the values of the constant $\alpha_{y_{g s j}^{*}}$ are the same for all the strategies $j$ which branch from the vertex located immediately after the edge representing $y_{g s i}^{*}$, and 2) that we have

$$
\left.\frac{\partial}{\partial \theta}\left[\log \frac{\partial}{\partial \theta} \Psi(\theta)\right\}\right]<0
$$

almost everywhere in the domain of $\theta$. To prove this, we obtain from $(2.2),(3.2),(3.4)$ and the definition of the basic function $A_{y_{g s i}^{\star}}(\theta)$, which was given by $(2.5)$, 


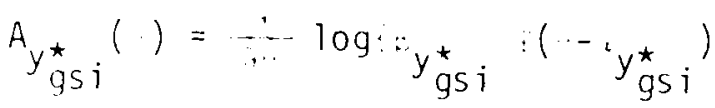

$\therefore$

$$
j^{\star} \ddot{y}_{g(s+1) j}^{*}\left(-{ }^{*} y_{g}^{\star}(s+1) j\right) ;
$$

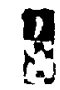

where $s$ indicates the summation over all the strategies $j$ branching from the vertex which lies immediately after the edge representing the union $y_{g s i}^{\star}$ in the differential strategy tree. By virtue of the first condition, we can rewrite (3.6) in the form

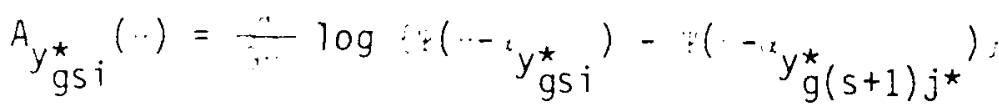

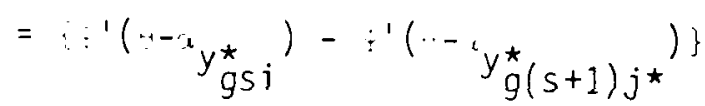

$$
\begin{aligned}
& \left\{\left(\cdots-y_{g s i}^{\star}\right)-\left(\cdots-y_{g(s+1)}^{\star} j^{\star}\right)\right\}^{-1} \text {, }
\end{aligned}
$$

where ${ }^{*}{ }^{\star}(s+1) j^{\star}$ indicate the common constant for all $j^{*}$ 's, and $\Psi^{\prime}(\cdot)$ indicates the first derivative of $\Psi^{(\cdot)}$ with respect to $A$. We notice that, if we replace $y_{g s i}^{*}$ by the graded item score $x_{g}$ and use $\left(\because-\alpha_{x_{g}}\right)$ as the cumulative operating characteristic $P_{x_{g}^{*}}(\vartheta)$, the last form of (3.7) is identical with the basic function of the graded item score, and the left hand side of (3.5) is identical with the corresponding asymptotic basic function. Thus we can say that all the unions, $y_{g s i}^{*}$, are equivalent to syndrome response categories (cf. Samejima, 1972, Section 5.2), and a unique maximum is assured for every possible response pattern.

8

$\Gamma_{0}$ 
IV. Information Provided by Differential Strategies

The information function, $I_{y_{g s i}^{*}}(A)$, for the union of the attainment scores $y_{g s i}^{*}$ is defined by

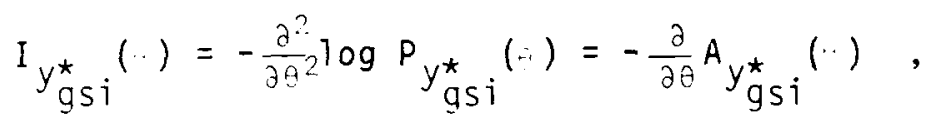

where $P_{y_{g s i}^{\star}}(\cdots)$ is the operating characteristic, and $A_{y_{g s i}^{\star}}($ (.) is the basic function, of $y_{g S i}^{*}$, which are defined by (2.2) and (2.5), respectively. This function is non-negative whenever the unique maximum condition is satisfied.

In the homogeneous case, as we observed in the preceding section, if there is a single value $\alpha_{y_{g s}^{*}}$ common for all the strategies $j^{\star}$ 's , which leads to the satisfaction of the unique maximum condition, we can write for the operating characteristics of $y_{\text {gs }}^{\star}$

$$
P_{y_{g s i}^{*}}(s)=z_{y_{g s i}^{\star}}\left[\Psi\left(\theta-\alpha_{y_{g s i}^{\star}}\right)-\Psi\left(\theta-\alpha_{y_{g}^{*}(s+1) j^{*}}\right)\right],
$$

which is obvious from (2.2), (3.2) and (3.4). Substituting (4.2) into (4.1) we obtain

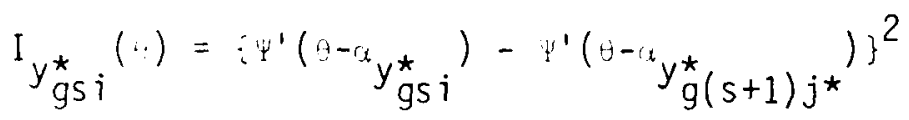

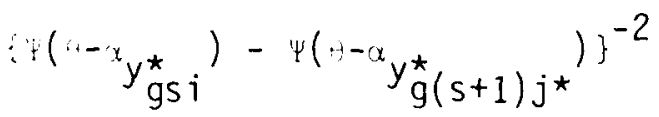




$$
\begin{aligned}
& -\left\{:\left(\theta-\alpha_{\left.y_{g s i}^{\star}\right)}\right)-\psi^{\prime \prime}\left(\theta-\alpha_{y_{g}^{\star}(s+1) j_{\star}}\right)\right\} \\
& \quad\left\{\psi\left(s-\alpha_{y_{g s i}^{\star}}\right)-\psi\left(\theta-\alpha_{y_{g}^{\star}(s+1) j^{\star}}\right)\right\}^{-1},
\end{aligned}
$$

where :"(•) indicates the second derivative of $\Psi(\cdot)$ with respect to 3 . From this result and (3.3), it is obvious that when $\theta$ approaches negative infinity $I_{y_{g s i}^{\star}}(\theta)$ tends to the limiting case of

$$
\begin{aligned}
\left\{y^{\prime \prime}\left(\theta-\alpha_{y_{g s i}^{\star}}\right)\right\}^{2} & \left\{\psi^{\prime}\left(\theta-\alpha_{y_{g s i}^{\star}}\right)\right\}^{-2} \\
& -\left\{y^{*}\left(\theta-\alpha_{\left.y_{g s i}^{\star}\right)}\right)\right\}\left\{\Psi^{\prime}\left(\theta-\alpha_{y_{g s i}^{*}}\right)\right\}^{-1}
\end{aligned}
$$

for $s=1,2, \ldots, m_{g i}$, where $\psi^{*}(\cdot)$ indicates the third derivative of

$(\cdot)$ with respect to $\theta$. It is also obvious that, when $\theta$ approaches positive infinity, the asymptote of $I_{y_{g s i}^{*}}(\theta)$ is also given as the limiting case of $(4.4)$ for $s=0,1,2, \ldots,\left(m_{g i}-1\right)$. Note that the above does not include the case where $s=0$ and $\theta$ approaches negative infinity, or the case where $s=m_{g i}$ and $\theta$ tends to positive infinity. In these two cases the asymptotes are obtained more straightforwardly from $(4.3)$

We can also see from (4.3) that this function is symmetric with $\hat{\theta}=\left[a_{y_{g s i}^{\star}}+a_{y_{g}^{*}(s+1) j^{\star}}\right] / 2$ as the axis of symmetry for $s=1,2, \ldots,\left(m_{g i}-1\right)$, wi enever $\psi(\cdot)$ is point-symmetric. At this point

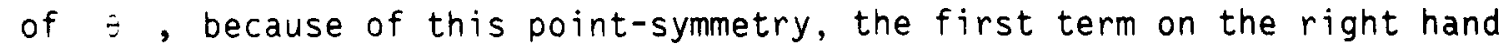
side of (4.3) disappears, and we have 


$$
\begin{aligned}
& I_{y_{g s i}^{\star}}(\theta)=2 y^{\prime \prime}\left\{\left(\alpha_{y_{g}^{\star}(s+1) j^{*}}{ }^{-\alpha} y_{g s i}^{\star}\right) / 2\right\} \\
& {\left[1-2 \psi\left\{\left(\alpha_{y_{g s i}^{\star}{ }^{-\alpha} y_{g}^{\star}(s+1) j \star}\right) / 2\right\}\right]^{-1},} \\
& \text { for } \theta=\left(\alpha_{y_{g s i}^{\star}}+\alpha_{y_{g}^{\star}(s+1) j \star}\right) / 2 .
\end{aligned}
$$

It has been shown that if $\Psi(\cdot)$ is either a normal ogive function or a logistic distribution function, which are defined by

$$
\Psi(t)=(2 \pi)^{-1 / 2} \int_{-\infty}^{a t} e^{-u^{2} / 2} d u
$$

and

$$
\Psi(t)=[1+\exp \{-D a t\}]^{-1},
$$

respectively, where $a(>0)$ is a constant and $D(>0)$ is a scaling factor, the unique maximum condition is satisfied (cf. Samejima, 1972, Section 5.2$)$. Note that in both models $\Psi(\cdot)$ is point-symmetric, and, therefore, $I_{y_{g s i}^{*}}(\theta)$ is symmetric for $s=1,2, \ldots,\left(m_{g i}-1\right)$.

For the item information function $I_{g}(\theta)$ we can write

$$
I_{g}(\theta)=E\left[I_{y_{g s i}^{*}}(\theta) \mid \theta\right]=\sum_{y_{g s i}^{\star}}^{\Sigma} I_{y_{g s i}^{*}}(\theta) P_{y_{g s i}^{*}}(\theta)
$$




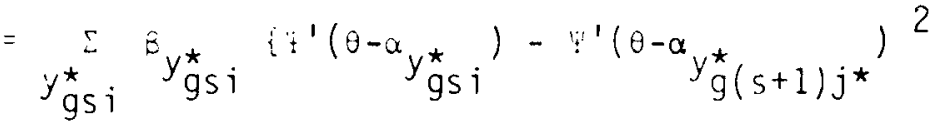

$$
\begin{aligned}
& \left\{\Psi\left(\theta-\alpha_{y_{g s i}^{\star}}\right)-\psi\left(\theta-\alpha_{y_{a}^{\star}(s+1) j \star}\right)\right\}^{-1}
\end{aligned}
$$

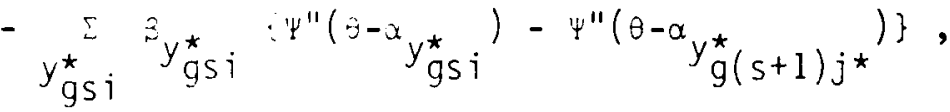

5

S.

where $y_{j}^{\Sigma}$ indicates the summation over all the unions of attainment scores, or over all the edges in the differential strategy tree. Since we have

$$
y_{g s i}^{\star \Sigma} P_{g s i}^{\star}(\theta)=1
$$

for the entire range of $\theta$, from this and (4.2) it is obvious that the second term of the rightest hand side of (4.8) equals zero. We obtain, therefore,

$$
\begin{aligned}
& I_{g}(\theta)=\sum_{y_{g s i}^{\star}}^{\sum} \beta_{y_{g s i}^{\star}}\left\{\Psi^{\prime}\left(\theta-\alpha_{y_{g s i}^{*}}\right)-\psi^{\prime}\left(\theta-\alpha_{y_{g}^{*}(s+1) j *}\right)\right\}^{2} \\
& \left\{\psi\left(\theta-\alpha_{y_{g s i}^{\star}}\right)-\psi\left(\theta-\alpha_{y_{g}^{\star}(s+1) j^{\star}}\right)\right\}^{-1} \text {. }
\end{aligned}
$$

It is obvious from (4.10) that within each strategy the more subprocesses we have the greater amount of item information we get, with the case of continuous subprocesses as the limiting situation. This can be observed by following a similar logic that is applied for the relationship between the graded response model and the continuous response mode 1 (Samejima, 1973). 
A question may arise as to the possibility of increasing the amount of item information by increasing the number of successful strategies. The answer is not directly positive, however. We can see it through the following example. Figure 4-1 presents the digraph and the differential strategy tree of a simple case where we have only two successful strategies. Suppose that the homogeneous case is applied and that we have a common value $\alpha_{y_{g l j}^{\star}}$. If, in addition, we have

$$
\left\{\begin{array}{l}
\alpha_{y_{g 21}^{*}}=\alpha_{y_{g 22}^{*}} \\
\alpha_{y_{g}^{*} 1}=\alpha_{y_{g}^{*} 32}
\end{array},\right.
$$

then the information function, which is given by (4.3), becomes identical for $i=1,2$ for the same attainment score $s(=0,1,2,3)$. From this fact and (3.4) we obtain from (4.10) for the item information function

$$
\begin{aligned}
& I_{g}(\theta)=\sum_{y_{g s 1}^{*}}\left\{\psi^{\prime}\left(\theta-\alpha_{\left.y_{g s}^{*}\right)}\right)-\psi^{\prime}\left(\theta-\alpha_{y_{g(s+1}^{*}}\right)\right\}^{2} \\
& \left\{\Psi\left(\theta-\alpha_{y_{g s 1}^{\star}}\right)-\Psi\left(\theta-\alpha_{y_{g}^{\star}(s+1) 1}\right)\right\}^{-1} \\
& =\sum_{g s 2}^{\sum}\left\{\psi^{\prime}\left(\theta-\alpha_{y_{g s 2}^{*}}\right)-\psi^{\prime}\left(\theta-\alpha_{y_{g}^{*}(s+1) 2}\right)\right\}^{2} \\
& \left\{\Psi\left(\theta-\alpha_{y_{g s 2}^{\star}}\right)-\Psi\left(e-\alpha_{y_{g}^{\star}(s+1) 2}\right)\right\}^{-1} \text {. }
\end{aligned}
$$

The amount of item information is, therefore, the sami as that of each single strategy case. Thus branching itself does not necessarily increase the amount of item information. 


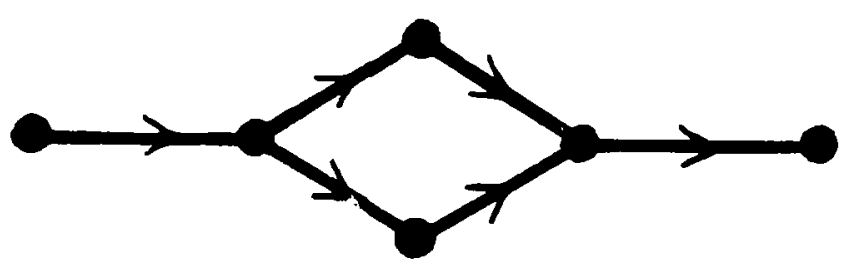

Bis

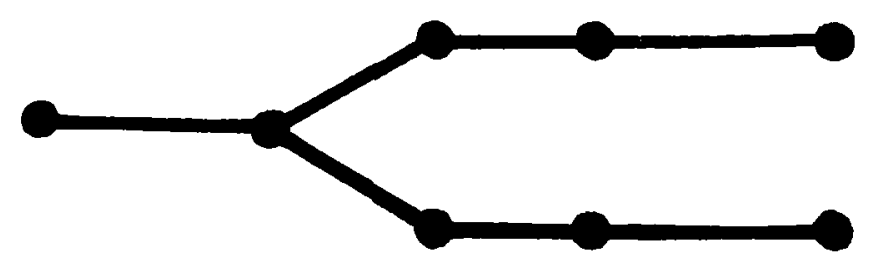

0

1

2

3

FIGURE 4-1

The Digraph and the Differential Strategy Tree of a Simple Case Where We Have Two Strategies and There Are Four Subprocesses in Each Strategy.

It is also obvious, however, that the differentiation of successful strategies contributes to the increment in the amount of item information through the varieties of different configurations of $P_{y_{g s i}^{*}}^{*}(\theta)$ across the separate strategies, for the more differential strategies we have the greater possibility there is that "detoured" strategies provide us with

r. greater numbers of subprocesses.

2

Tis 
When we have $n$ problem solving tasks which require the same latent trait $t$, we can write for the test information function $I(\theta)$

$$
I(\theta)=\sum_{g=1}^{n} I_{g}(\theta) \text {, }
$$

following a similar logic applied for the graded response model.

In the normal ogive model, from (3.2) and (4.6) we can write for the cumulative operating characteristic of $y_{g s i}^{*}$

$$
P_{y_{g s i}^{*}}^{*}(\theta)=\beta_{y_{g S i}^{*}}(2 \pi)^{-1 / 2} \int_{-\infty}^{-a_{g}\left(\theta-b_{y_{g s i}^{*}}\right)} e^{-u^{2} / 2} d u,
$$

where $a_{g}(>0)$ is the item discrimination parameter, and $b_{y_{g s i}^{*}}$ is the difficulty parameter for the subprocess $y_{\text {gsi }}^{*}$, which replaces $\alpha_{y_{g s i}^{*}}$ by virtue of the familiarity in notation. Thus we have for the first and second derivatives of $\mathrm{P}_{\mathrm{gsi}}^{*}(\theta)$ with respect to $\theta$

$$
p_{y_{g s i}^{*}}^{* \prime}(\theta)=\beta_{y_{g s i}^{*}}(2 \pi)^{-1 / 2} a_{g} \exp \left[-a_{g}^{2}\left(\theta-b_{y_{g s i}^{*}}\right)^{2} / 2\right]
$$

and

$$
p_{g s i}^{* \prime \prime}(\theta)=-a_{g}^{2}\left(\theta-b_{y_{g s i}^{*}}\right) p_{y_{g s i}^{*}}^{*}(\theta),
$$

or, alternatively, from (4.6) we can write

$$
\Psi^{\prime}\left(\theta-b_{y_{g s i}^{\star}}\right)=(2 \pi)^{-1 / 2} a_{g} \exp \left[-a_{g}^{2}\left(\theta-b_{y_{g s i}^{*}}\right)^{2} / 2\right]
$$


and

$$
y^{\prime \prime}\left(\hat{v}-b_{y_{g s i}^{*}}\right)=-a_{g}^{2}\left(\theta-b_{y_{g s i}^{*}}\right) \Psi^{\prime}\left(\theta-b_{y_{g s i}^{*}}\right) .
$$

Figure 4-2 presents the cumulative operating characteristics of the unions of attainment scores, which follow the normal ogive model, of a relatively simple example which was given as Example 1 in the preceding research report. In this example, $a_{g}=1.00, b_{y_{g 11}^{*}}=-2.50$,

$$
\begin{aligned}
& b_{y^{\prime} \star}=-1.00, \quad b_{y_{g}^{\star} 1}=0.50, b_{y_{g 22}^{\star}}=-1.80, \quad b_{y_{g 32}^{\star}}=0.00, \\
& b_{y_{g 42}^{\star}}=2.00, \beta_{y_{g 11}^{\star}}=0.60 \text { and } \beta_{y_{g}^{*} 12}=0.40 \text {. The corresponding }
\end{aligned}
$$
operating characteristics for these unions are presented in Figure $4-3$. The differential strategy tree of this example was presented in the preceding research report, and a?:jo here as figure 4-4. Since there are as many as eight edges in the differential strategy tree, each edge is drawn by a unique type of line. These separate types of lines will be carried to the subsequent two figures for the sake of identification, so that we shall be able to avoid confusions.

The corresponding eight information functions $\mathrm{I}_{\mathrm{y}_{\mathrm{gsi}}^{\star}}(\theta)$ are presented in Figure 4-5, together with the item information function which is shown by a solid line. Since in the normal ogive model we have

$$
\psi^{\bullet}\left(\theta-b_{y_{g s i}^{*}}\right)=a_{g}{ }^{2} \psi^{\bullet}\left(\theta-b_{y_{g s i}^{*}}\right)\left[a_{g}{ }^{2}\left(\theta-b_{y_{g s i}^{*}}\right)^{2}-1\right],
$$

which is obvious from (4.18), we obtain

$$
y^{\prime \prime}\left(\theta-b_{y_{g s i}^{*}}\right) / \psi^{\prime}\left(\theta-b_{y_{g s i}^{*}}\right)=-a_{g}^{2}\left(\theta-b_{y_{g s i}^{*}}\right),
$$



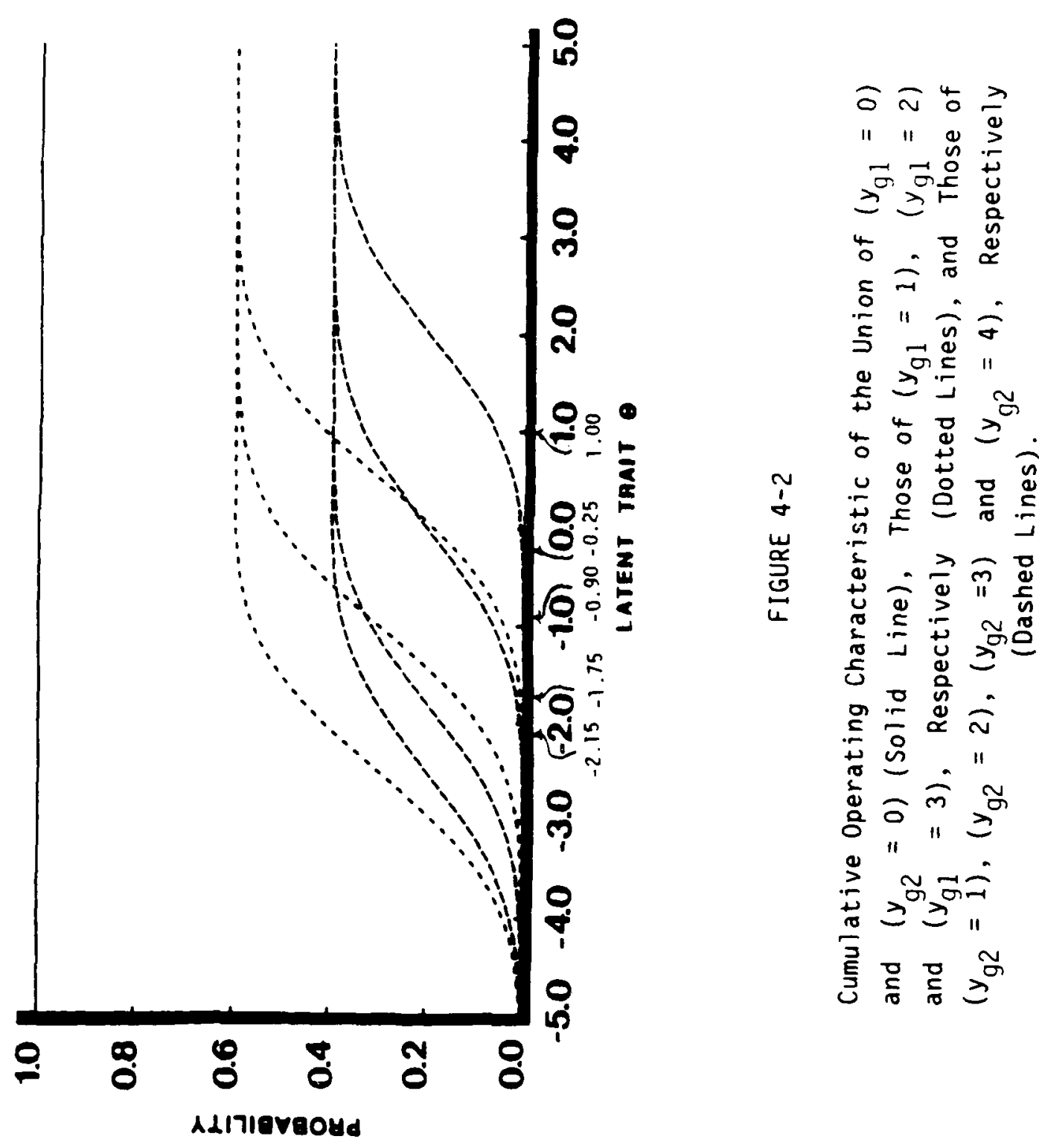
E

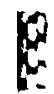

1

6

5

6
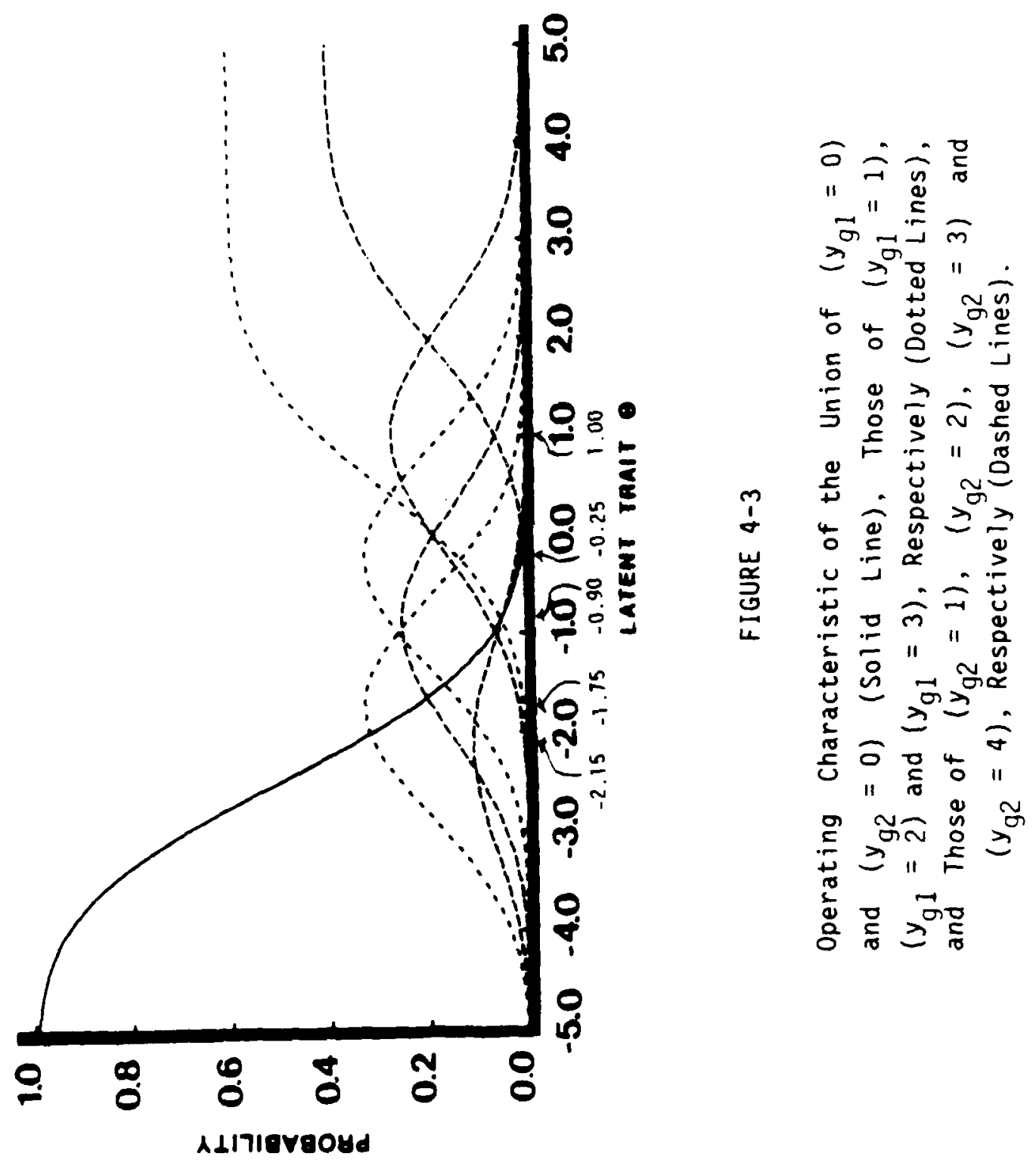


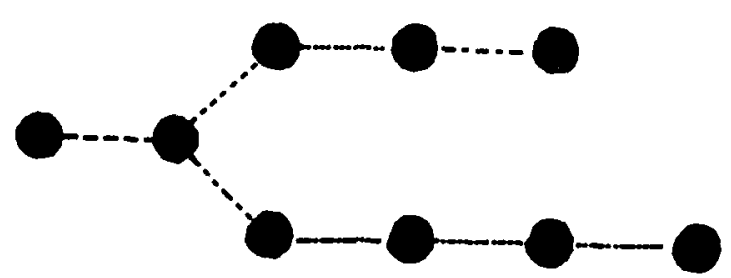

FIGURE 4-4

Differential Strategy Tree Whose Edges Are Drawn by the Same Separate Types of Lines As Those in Figures $4-5$ and 4-6.

$$
\psi^{*}\left(\theta-b_{y_{g s i}^{\star}}\right) / \psi^{\prime}\left(\theta-b_{y_{g s i}^{\star}}\right)=a_{g}{ }^{2}\left[a_{g}{ }^{2}\left(\theta-b_{y_{g s i}^{*}}\right)-1\right],
$$

and finally

$$
\left\{\begin{array}{l}
\lim _{\theta \rightarrow-\infty} I_{y_{g s i}^{\star}}(\theta)=a_{g}{ }^{2} \text { for } s=1,2,3, \ldots, m_{g i} \\
\lim _{\theta \rightarrow \infty} I_{y_{g s i}^{\star}}(\theta)=a_{g}{ }^{2} \text { for } s=0,1,2, \ldots,\left(m_{g i}-1\right) .
\end{array}\right.
$$

Note that this common asymptotic value equals the constant item response information function of the continuous item response in the normal ogive model ( $c f$. Samejima, 1973). Since in our example a $=1.00$, these asymptotes are unity, as we can see in Figure $4-5$. When $s=0$, since we have 
th

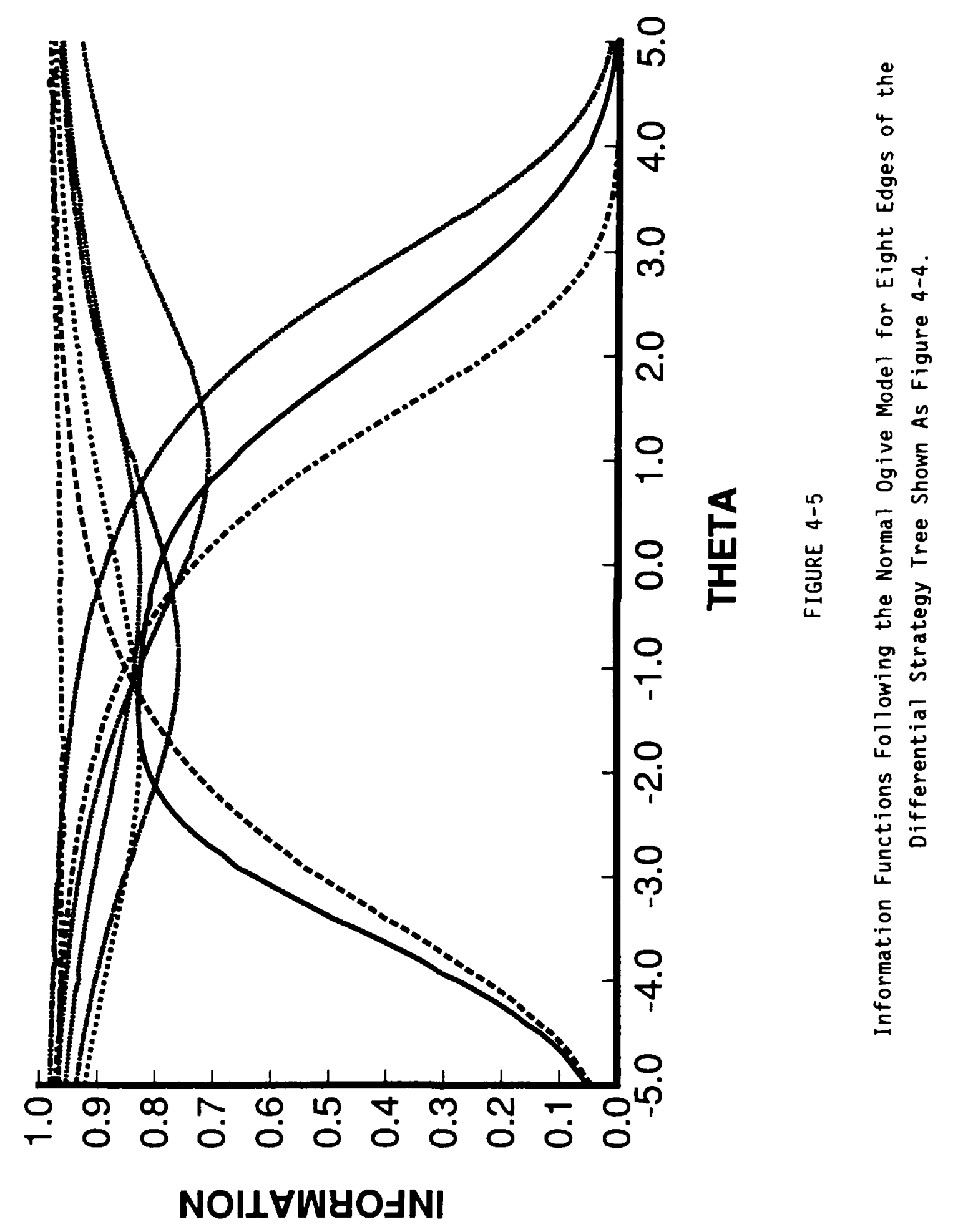

8

5 
(4.23)

$$
\left\{\begin{array}{l}
\psi\left(\theta-b_{y_{g 0 i}^{\star}}\right)=1 \\
\psi^{\prime}\left(\theta-b_{y_{g 0 i}^{\star}}\right)=\psi^{\prime \prime}\left(\theta-b_{y_{g 0 i}^{\star}}\right)=0,
\end{array}\right.
$$

from this and (4.3) we can write

$$
\begin{aligned}
I_{y_{g 0 i}^{*}}(\theta)=\left\{\Psi^{\prime}\left(0-b_{y_{g l i}^{*}}\right)\right\}^{2}\left\{1-\Psi\left(\theta-b_{y_{g}^{*} 1 i}\right)\right\}^{-2} & \\
& +\left\{\Psi^{\prime \prime}\left(\theta-b_{y_{g l i}^{\star}}\right)\right\}\left\{1-\psi\left(\theta-b_{y_{g}^{*}}\right)\right\}^{-1},
\end{aligned}
$$

and, therefore, we obtain

$$
\lim _{\theta \rightarrow-\infty} I_{y_{\mathrm{g} 0 i}^{\star}}(\theta)=0
$$

When $s=m_{g i}$, since we can write

$$
\begin{gathered}
\Psi\left(\theta-b_{y_{g}^{*}\left(m_{g i}+1\right) i}\right)=\psi^{\prime}\left(\theta-b_{y_{g}^{*}\left(m_{g i}+1\right) i}\right) \\
=\psi^{\prime \prime}\left(\theta-b_{y_{g}^{*}\left(m_{g i}+1\right) i}\right)=0,
\end{gathered}
$$

substituting this into (4.3) we have 


$$
\begin{aligned}
& I_{y_{g m_{g i}}^{\star} i}(e)=\left\{\Psi^{\prime}\left(\theta-b_{y_{g m_{g i}}^{\star} i}\right)\right\}^{2}\left\{\psi\left(\theta-b_{y_{g m}^{\star}}\right)\right\}^{-2} \\
& -\left\{\Psi ^ { \prime \prime } \left(\theta-b_{y_{g m}^{*}} j i\right.\right.
\end{aligned}
$$

and, therefore, we obtain

$$
\lim _{\theta \rightarrow \infty} I_{y_{g m_{g i} i}^{\star}}(\theta)=0
$$

These asymptotes are shown in the three curves of Figure 4-5.

As was observed earlier, $I_{y_{g s i}^{\star}}(\theta)$ is symmetric in the normal ogive model as $\theta=\left[b_{y_{g s i}^{\star}}+b_{y_{g}^{*}(s+1) j^{\star}}\right] / 2$ as the axis of symmetry for $s=1,2,3, \ldots,\left(m_{g i}-1\right)$. From $(4.5)$ and $(4.18)$ we obtain

$$
\begin{aligned}
& I_{y_{g s i}^{*}}(\theta)=a_{g}{ }^{2}\left(b_{y_{g}^{*}(s+1) j *}{ }^{-b_{y_{g s i}^{*}}}\right) \\
& \Psi^{\prime}\left\{\left(b_{y_{g}^{\star}(s+1) j^{*}}-b_{y_{g s i}^{\star}}\right) / 2\right\} \\
& {\left[1-2 \Psi\left\{\left(b_{y_{g s i}^{*}}-b_{y_{g}^{*}(s+1) j *}\right) / 2\right\}\right]^{-1} \text {, }} \\
& \text { for } \theta=\left(b_{y_{g s i}^{*}}+b_{y_{g}^{*}(s+1) j^{\star}}\right) / 2 \text {. }
\end{aligned}
$$

We notice that this value decreases as the distance between $b_{v_{g s i}^{*}}$ and $b_{y^{\star}(s+1) j^{\star}}$ increases, with $a_{g}^{2}$ and zero as its two asymtotes.

Figure 4-6 presents the information share, $\operatorname{I}_{y_{g s i}^{\star}}(\theta) P_{y_{g s i}^{\star}}(\theta)$, for 


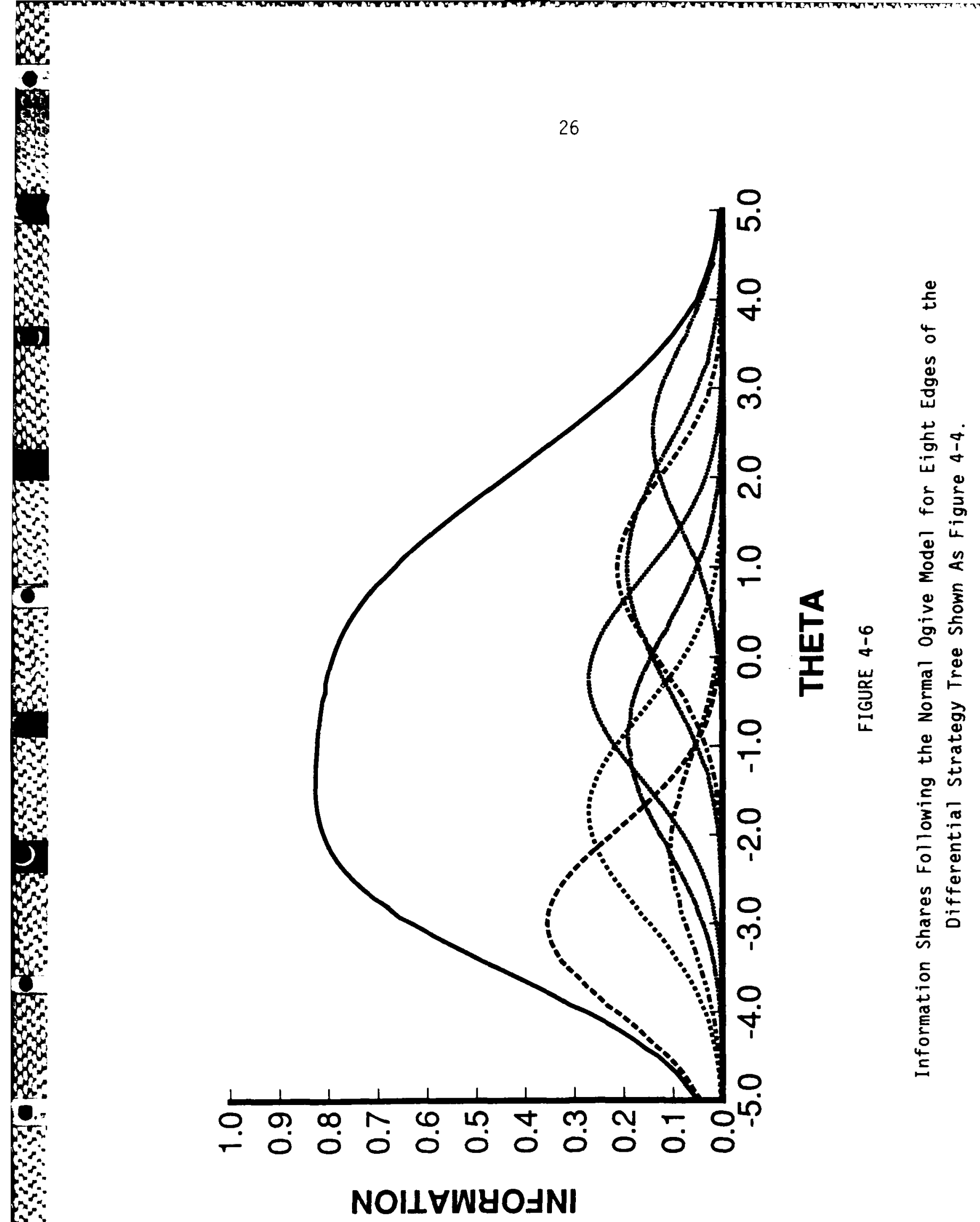


each of the eight $y_{g s i}^{*}$ 's, together with the item information function.

In the logistic model, as is obvious from (3.2) and (4.7), we have for the cumulative operating characteristic

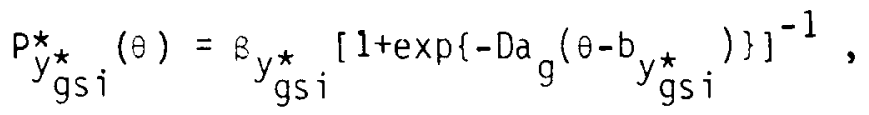

where $a_{g}$ and $b_{y_{g s i}^{*}}$ are the same parameters that are used in the normal ogive model, and $D(>0)$ is the scaling factor which is usually set equal to 1.7 in order to make the discrimination parameter $a_{g}$ comparable to the one in the normal ogive model. Thus we have for the first and second derivatives of $\mathrm{P}_{\mathrm{gsi}}^{*}(\theta)$ with respect to $\theta$

$$
\begin{aligned}
& P_{y_{g s i}^{*}}^{*^{\prime}}(\theta)=B_{y_{g s i}^{*}} D a g_{g}\left\{\exp \left\{-D a_{g}\left(\theta-b_{y_{g s i}^{*}}\right)\right\}\right. \\
& {\left[1+\exp \left\{-D a_{g}\left(\theta-b_{y_{g s i}^{*}}\right)\right\}\right]^{-2}}
\end{aligned}
$$

and

$$
P_{y_{g s i}^{* \prime \prime}}^{*}(\theta)=D a_{g} P_{y_{g s i}^{* \prime}}^{*}(\theta)\left[1-2\left\{\exp \left[-D a_{g}\left(\theta-b_{y_{g s i}^{*}}^{*}\right)\right]\right\}^{-1}\right],
$$

or we can write from $(4.7)$

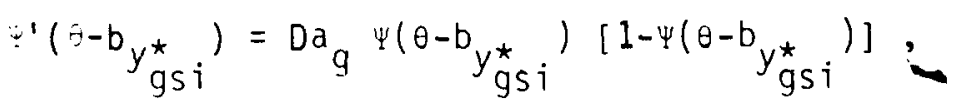

$$
\psi^{\prime \prime}\left(\theta-b_{y_{g s i}^{*}}\right)=D a_{g} \psi^{\prime}\left(\theta-b_{y_{g s i}^{*}}\right)\left[1-2 \Psi\left(\theta-b_{y_{g s i}^{*}}\right)\right],
$$

: 
and

$$
\begin{aligned}
\psi^{*}\left(j-b_{y_{g s i}^{*}}\right)=D^{2} a_{g}^{2} \psi^{\prime}\left(\theta-b_{y_{g s i}^{*}}\right) \\
\\
{\left[1-6 \psi\left(\theta-b_{y_{g s i}^{*}}\right)+6\left\{\psi\left(\theta-b_{y_{g s i}^{*}}\right)\right\}^{2}\right] . }
\end{aligned}
$$

From these results and (4.3) we can write

$$
I_{y_{g 0 i}^{*}}(\theta)=D^{2} a_{g}^{2} \Psi\left(\theta-b_{y_{g l i}^{*}}\right)\left[1-\Psi\left(\theta-b_{y_{g l i}^{*}}\right)\right]
$$

and

$$
I_{y_{g m g i}^{*} i}(\theta)=D^{2} a_{g}^{2} \Psi\left(\theta-b_{y_{g m}^{*}}{ }_{g i}^{i}\right)\left[1-\Psi\left(\theta-b_{y_{g m}^{*}{ }_{g i} i}\right)\right] .
$$

Thus unlike the normal ogive model this model provides us with symmetric and unimodal functions for $I_{y_{g 0 i}^{*}}(\theta)$ and $I_{y_{g m_{g i}}^{\star}}(\theta)$, with the modal points at $\theta=b_{y_{g l i}^{*}}$ and $\theta=b_{y_{g m_{g i} i}^{*}}$, respectively. At each of these modal points, the function assumes $D^{2} a_{g}{ }^{2 / 4}$.

As for the other edges, since we have from (4.33), (4.34) and (4.35)

$$
\psi^{\prime \prime}\left(\theta-b_{y_{g s i}^{*}}\right) / \psi^{\prime}\left(\theta-b_{y_{g s i}^{*}}\right)=D_{g}\left[1-2 \Psi\left(\theta-b_{y_{g s i}^{*}}\right)\right],
$$

and

$$
\begin{aligned}
& \psi *\left(\theta-b_{y_{g s i}^{\star}}\right) / \psi^{\prime}\left(\theta-b_{y_{g s i}^{\star}}\right) \\
& =D^{2} a_{g}{ }^{2}\left[1-6 \psi\left(\theta-b_{y_{g s i}^{\star}}\right)+6\left\{\psi\left(\theta-b_{y_{g s i}^{\star}}\right)\right\}^{2}\right],
\end{aligned}
$$


from (4.4) we can see that the asymptote of $I_{y_{g s i}^{*}}(\theta)$ is given as the limiting case of

$$
20^{2} a_{g}^{2} \Psi\left(\theta-b_{y_{g s i}^{\star}}\right)\left[1-\psi\left(\theta-b_{y_{g s i}^{\star}}\right)\right]
$$

for $s=1,2, \ldots,\left(m_{g i}-1\right)$, in either case where $\theta$ tends to negative or positive infinity. Thus again unlike the normal ogive model this model provides us with one or more modal points for $\operatorname{I}_{y_{g s i}^{\star}}(\theta)$, with zero as the two asymptotes when $\theta$ tends to negative and positive infinities, for $s=1,2, \ldots,\left(m_{g i}-1\right)$. For these edges, the axis of symmetry for $I_{y_{g s i}^{\star}}(\theta)$ lies at $\theta=\left(b_{y_{g s i}^{\star}}+b_{y_{g}^{\star}(s+1) j^{*}}\right) / 2$, and at this point of $\theta$

$$
\begin{aligned}
I_{y_{g s i}^{*}}(\theta)=20^{2} a_{g}^{2} \Psi\left\{\left(b_{y_{g}^{*}(s+1) j^{*}}-b_{y_{g s i}^{*}}\right) / 2\right\} \\
{\left[1-\Psi\left\{\left(b_{y_{g}^{*}(s+1) j^{*}}-b_{y_{g s i}^{*}}\right) / 2\right\}\right] . }
\end{aligned}
$$

Figures 4-7 and 4-8 present the cumulative operating characteristics and the operating characteristics of the eight edges of the differential strategy trees following the logistic model, respectively, with the scaling factor, $D=1.7$, and with the same parameter values that we used for the normal ogive model. We can see that these graphs are very similar to those on the normal ogive model, which were shown as figures $4-2$ and $4-3$. The corresponding information functions for $y_{g s i}^{*}$ and information shares are shown in Figures $4-9$ and 4-10, respectively, together with $I_{g}(\theta)$.

Figures 4-11 through 4-14 present these four sets of functions for the five strategy case illustrated in Figures $1-1$ and 1-2. They follow the normal ogive model, with the parameters, $a_{g}=1.0, b_{y_{g}^{*}}=-2.5$, $b_{y_{g 21}^{\star}}=-2.0, \quad b_{y_{g}^{\star} 31}=-1.5, \quad b_{y_{g 41}^{\star}}=0.5, \quad b_{y_{g 51}^{*}}=1.5$, 


$$
\begin{aligned}
& b_{y_{g 32}^{\star}}=-1.5, \quad b_{y_{g 42}^{\star}}=-0.5, b_{y_{g 52}^{\star}}=1.5, b_{y_{g 62}^{\star}}=2.5, \\
& b_{y_{g 13}^{\star}}=-2.5, \quad b_{y^{\star} 23}=-1.8, \quad b_{y_{g 33}^{\star}}=-0.3, \quad b_{y^{\star} 43}=1.8, \\
& b_{y_{g 14}^{\star}}=-2.5, \quad b_{y^{\star} 24}=-1.5, \quad b_{y^{\star} 34}=-1.0, \quad b_{y_{g 44}^{\star}}=0.0 \text {, } \\
& b_{y^{\star} 35}=-1.0, \quad b_{y}^{*}=0.7, \quad b_{y^{\star} 55}=2.0, \quad B_{y_{g 11}^{\star}}=0.400 \text {, } \\
& B_{y_{g 13}^{*}}=0.250, \quad B_{y_{g i 4}^{*}}=0.350, \quad B_{y_{g}^{*}}=0.160, \quad B_{y_{g}^{*} 2}=0.240, \\
& B_{y_{g}^{\star} 34}=0.210 \text { and } B_{y_{g 35}^{\star}}=0.140 \text {. The corresponding results for the }
\end{aligned}
$$

logistic model are shown as Figures 4-15 through 4-18. Note that in each of these figures five separate graphs were drawn for the five successful strategies, in order to avoid confusions by presenting too many curves in a single graph.

Comparison of these functions in the normal ogive model with those in the logistic model in each of the two examples reveals that, in spite of the similarity between the two models with respect to their cumulative operating characteristics, and, therefore, to the operating characteristics of $y_{g s i}^{\star}$, there exist substantial differences between the two models in terms of their information functions. These differences do not affect the information shares too much, however, except that those functions are steeper in the logistic model than in the normal ogive model. As the result, neither do they affect the item information function $\operatorname{I}_{g}(\theta)$ too much.

These are only two of the many conceivable mathematical models. Some models provide us with cumulative operating characteristics which are not point-symetric as those in the normal ogive and logistic models are. In such cases it is expected that those functions will be more complicated as the result of this asymmetry. 


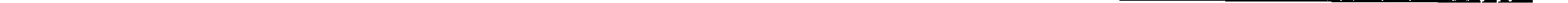




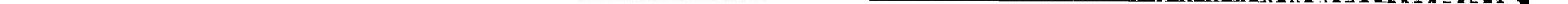




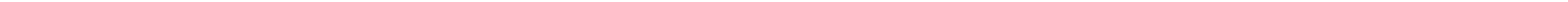



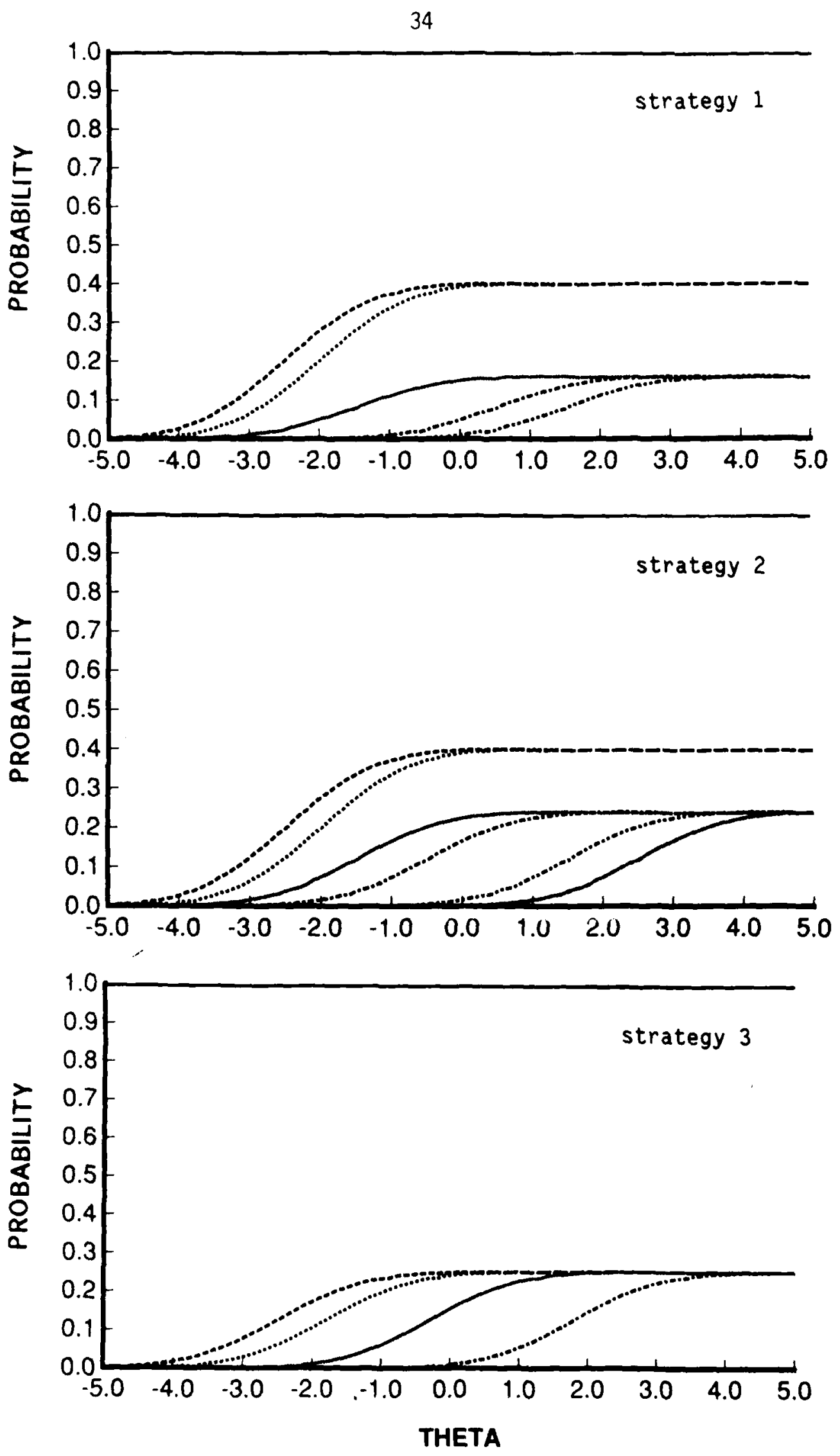

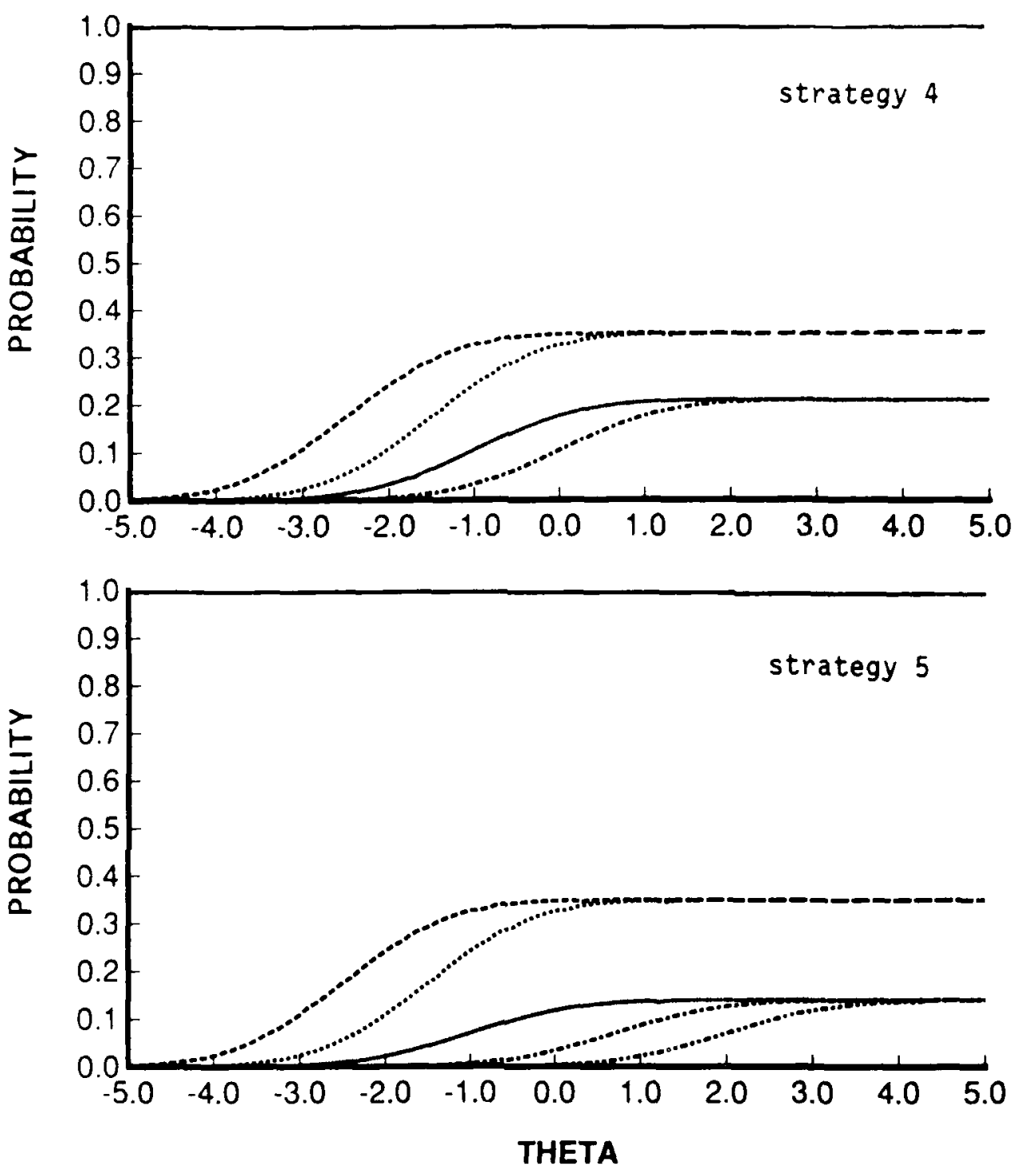

FIGURE $4-11$

Cumu?ative Operating Characteristics of the Subprocesses in E: $:$ h of the Five Successful Strategies of the Example Whose Differential Strategy Tree Is Shown as Figure 1-2, Following the Normal Ogive Model.

8

6 

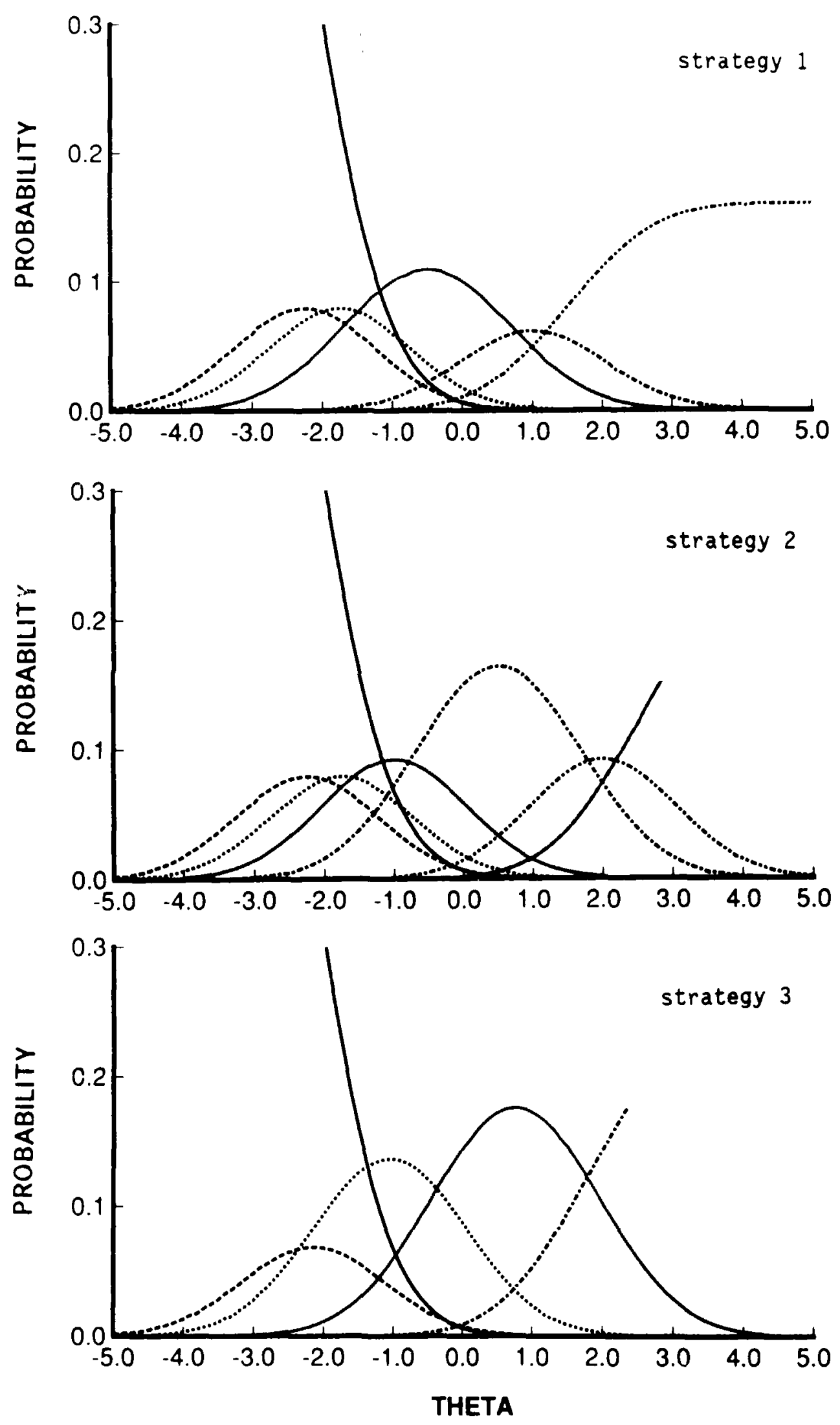

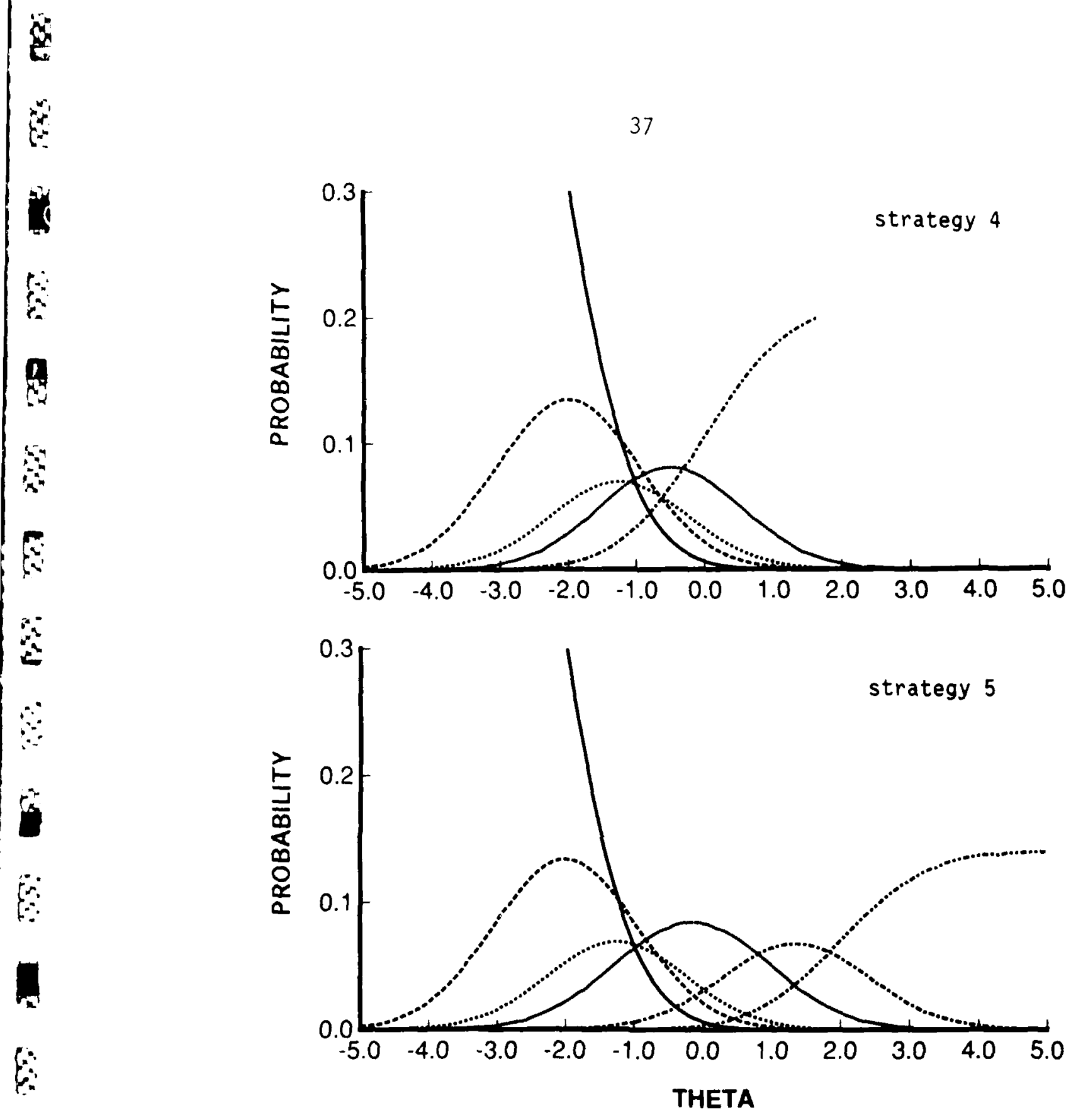

FIGURE $4-12$

Operating Characteristics of the Subprocesses in Each of the Five Successful Strategies of the Example Whose Differential Strategy Tree Is Shown as Figure 1-2,

i: Following the Normal Ogive Model.

$\therefore$

$\Gamma$ 


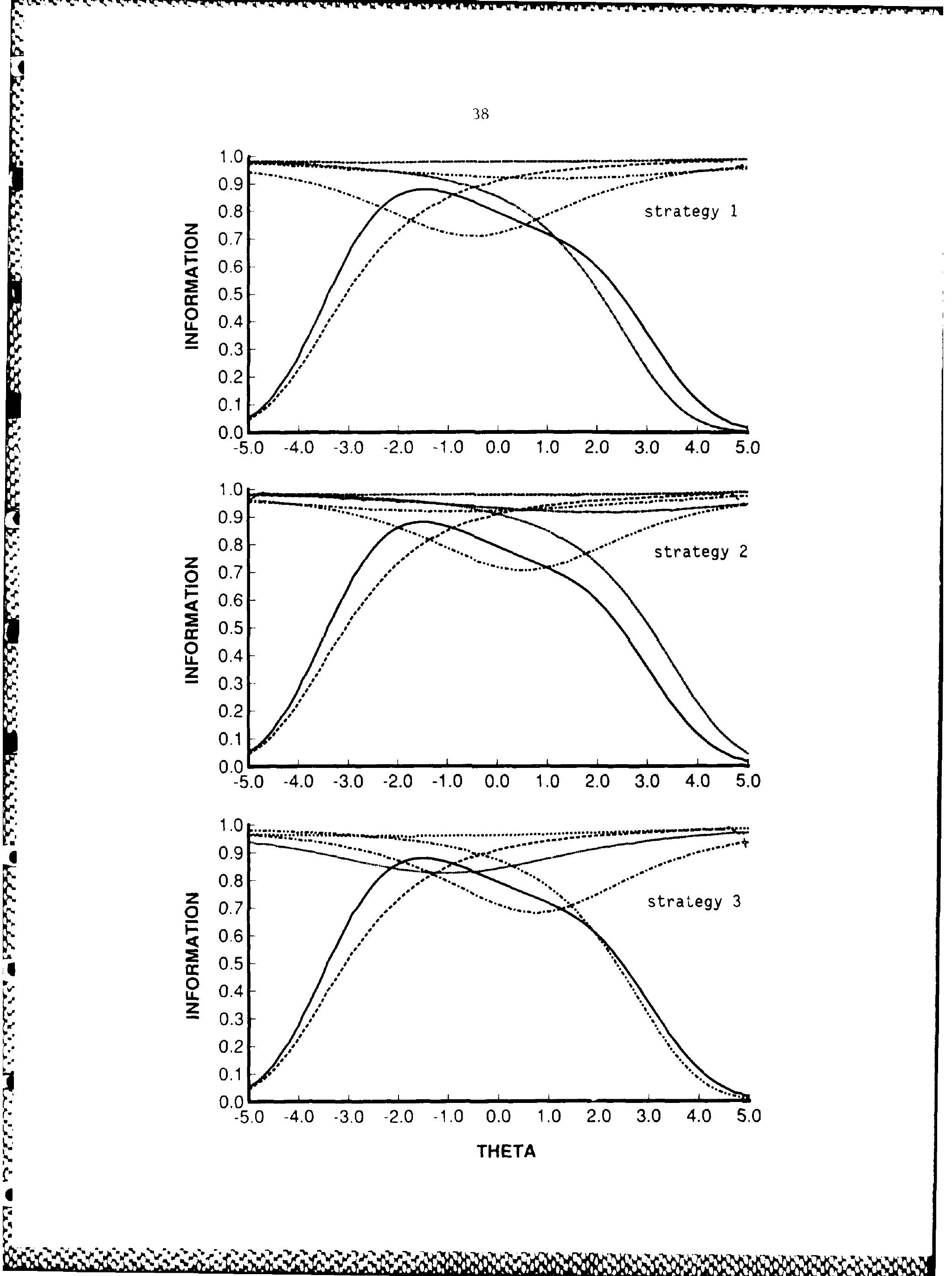



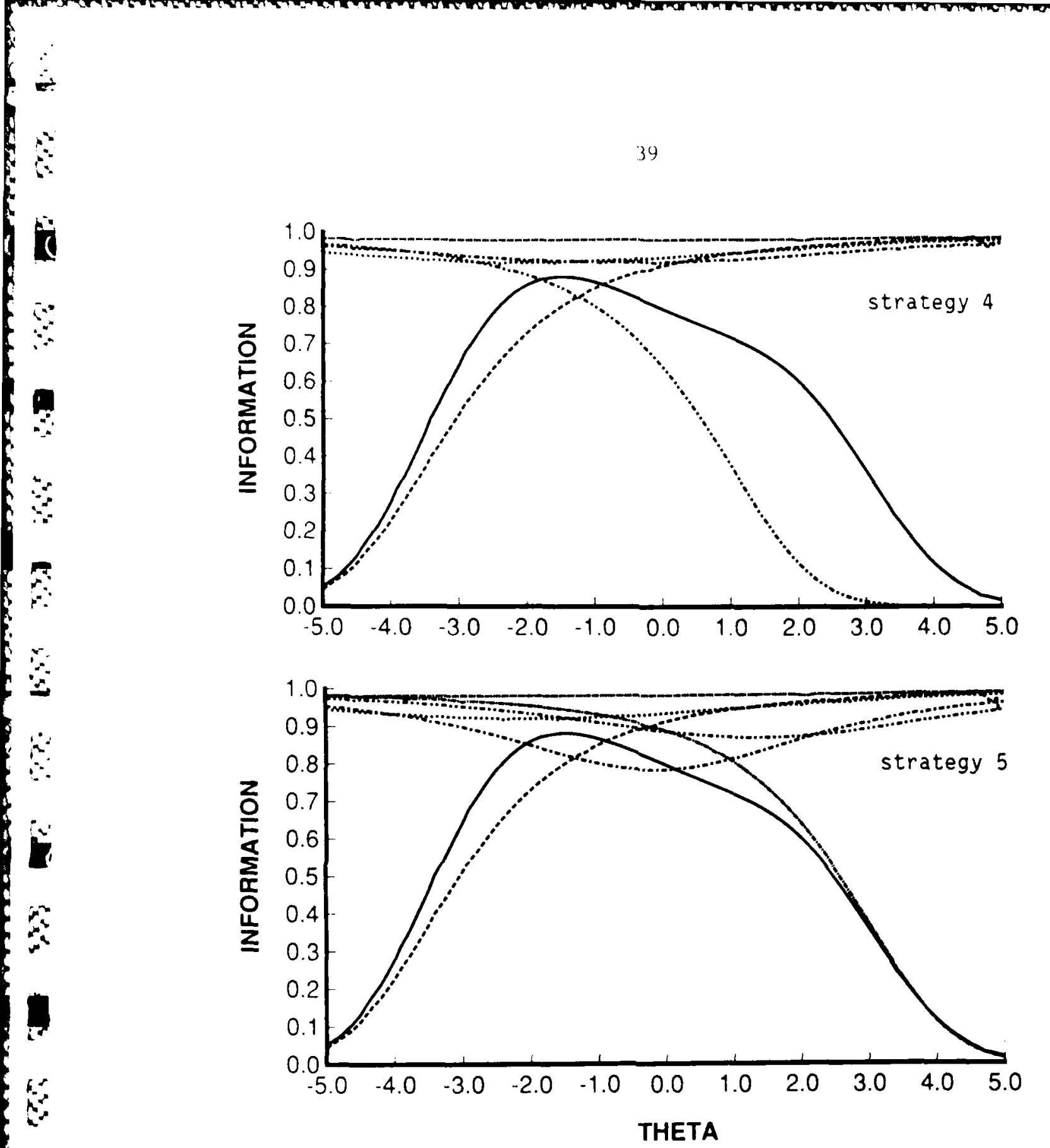

FIGURE $4-13$

Information Functions of the Subprocesses in Each of the

Five Successful Strategies of the Example Whose

Differential Strategy Tree is Shown as Figure 1-2,

Following the Normal Ogive Model. 

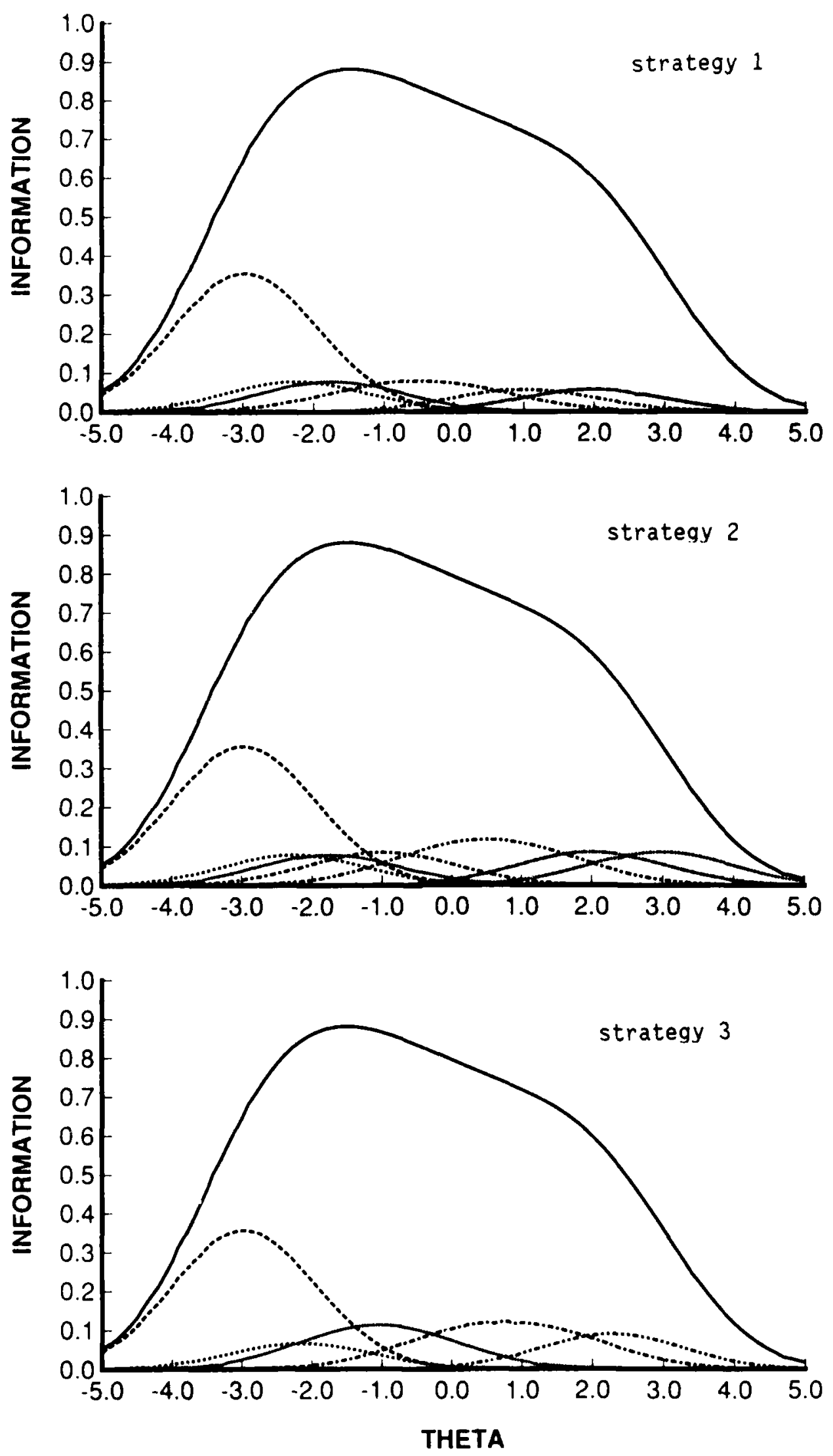

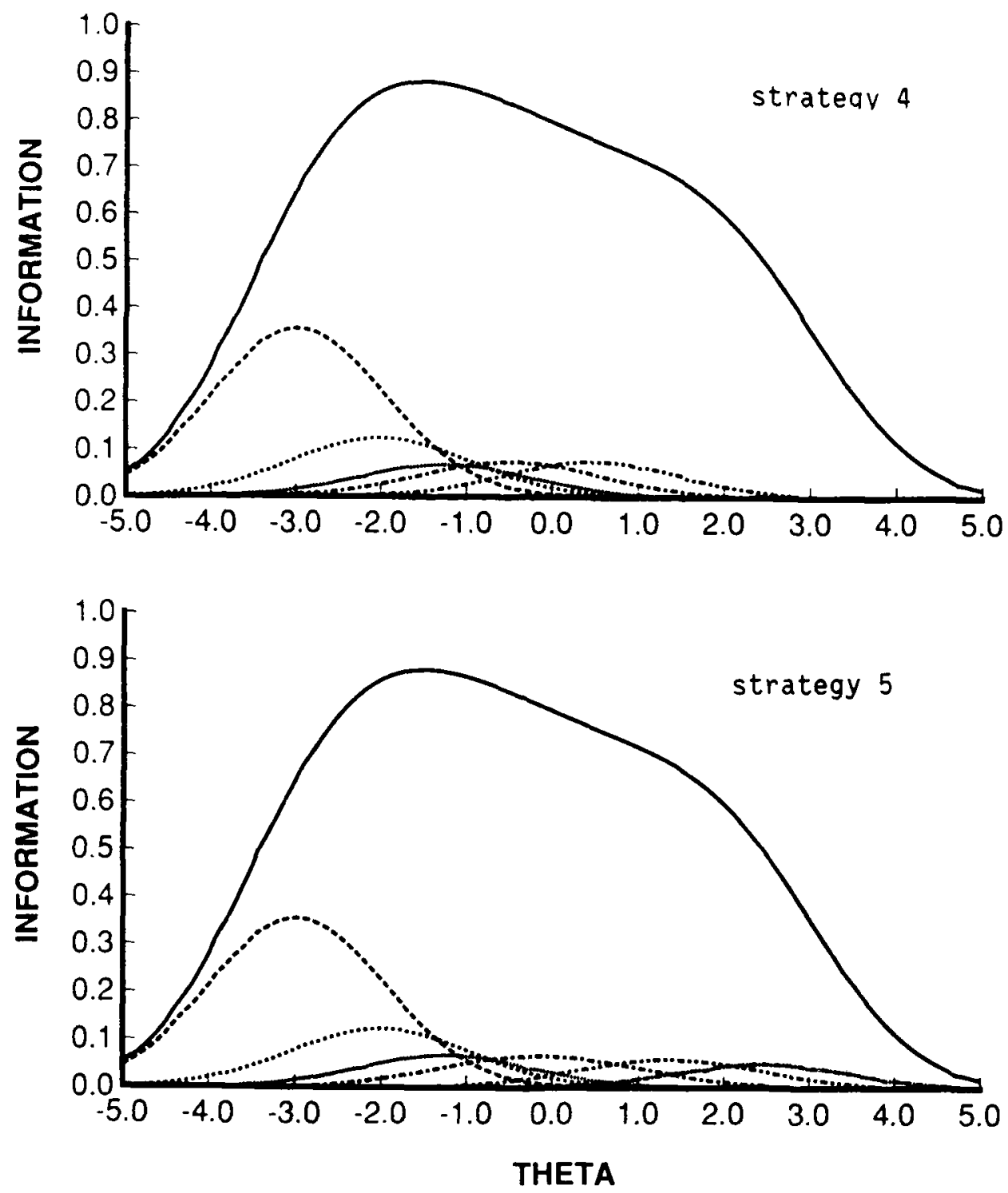

FIGURE $4-14$

Information Shares the Subprocesses in Each of the Five Successful Strategies of the Example Whose Differential Strategy Tree Is Shown as Figure 1-2, Following the Normal Ogive Model. 

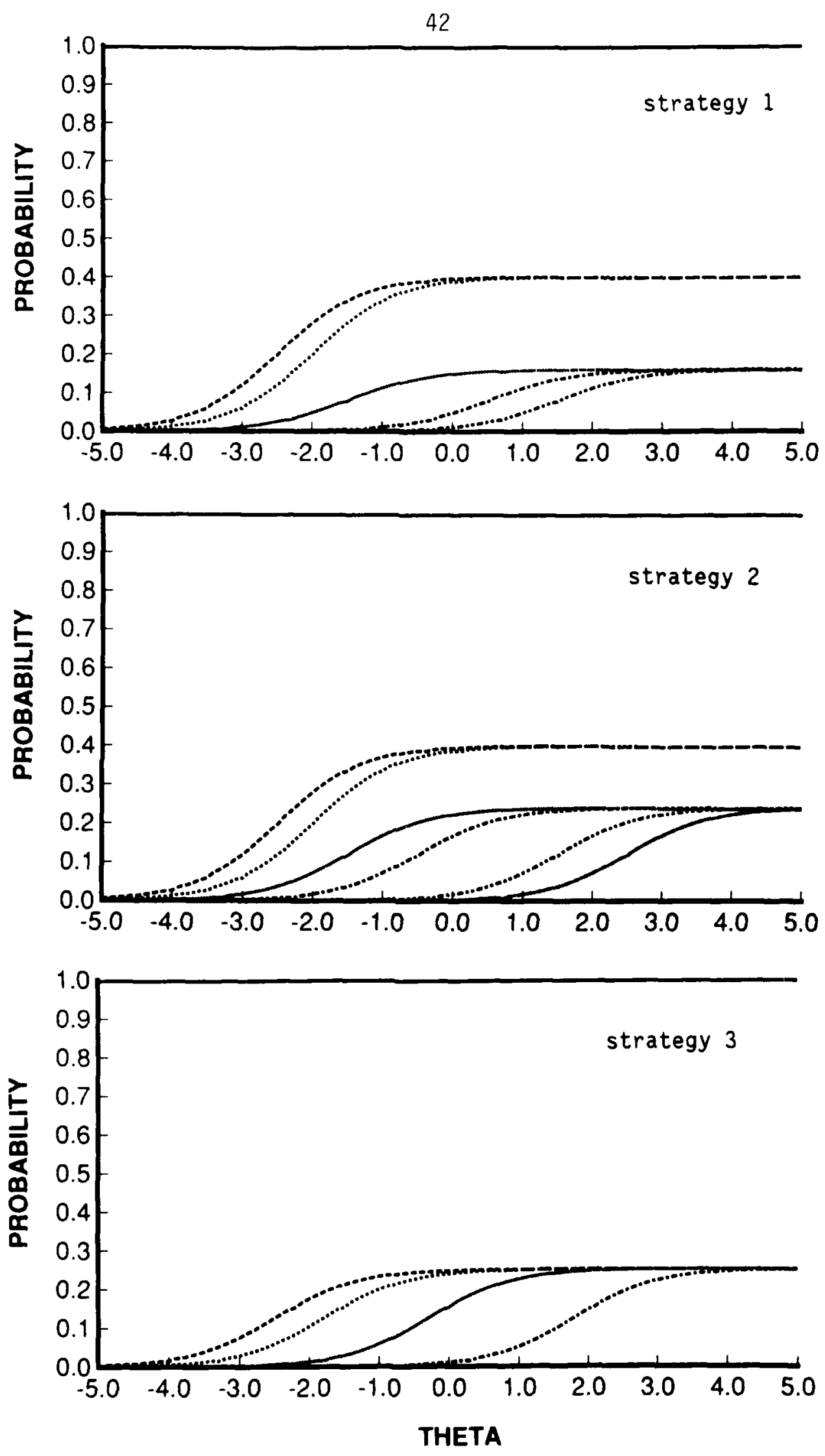

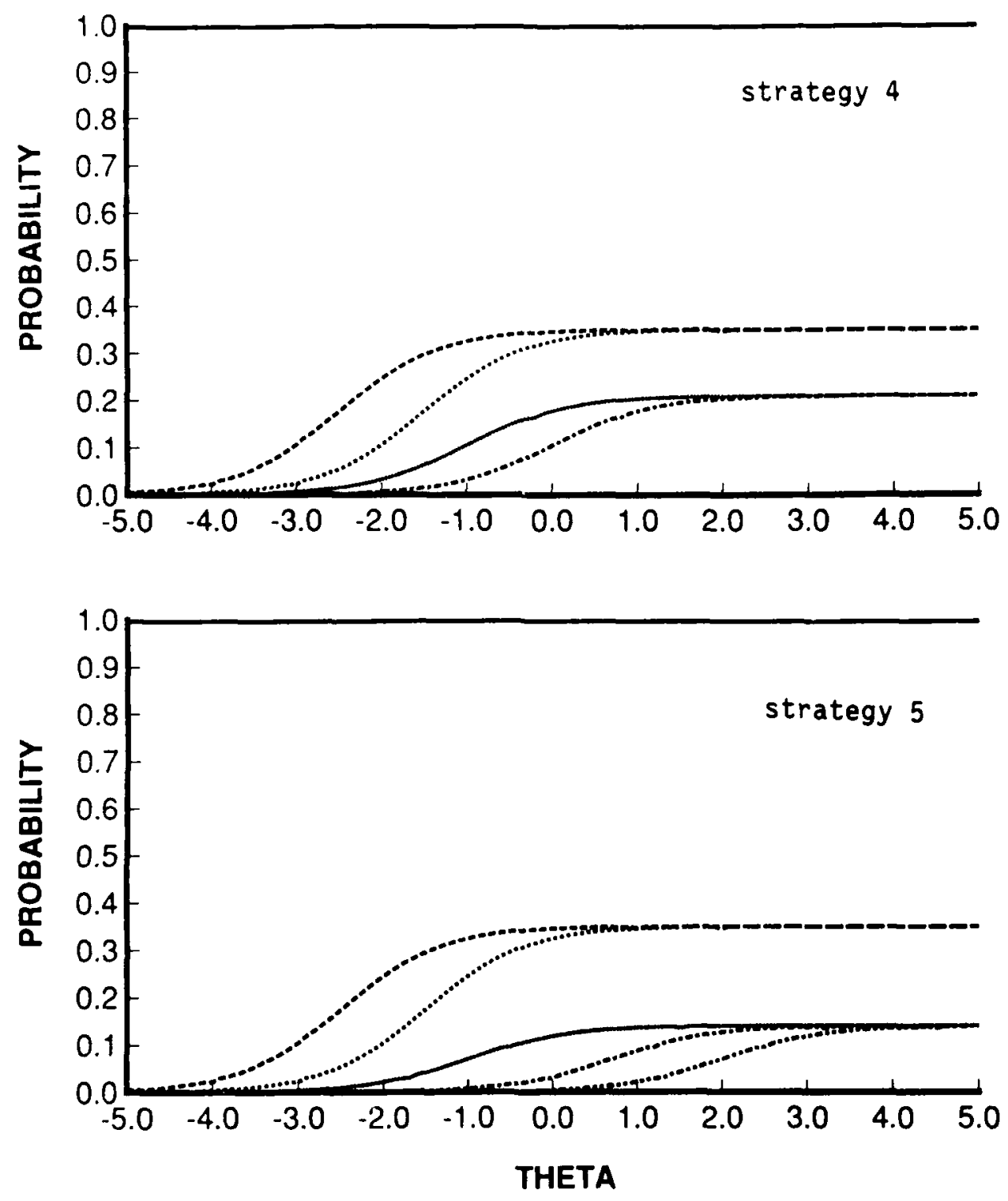

FIGURE $4-15$

Cumulative Operating Characteristics of the Subprocesses in Each of the Five Successful Strategies of the Example Whose Differential Strategy Tree Is Shown as Figure 1-2, Following the Logistic Model. 

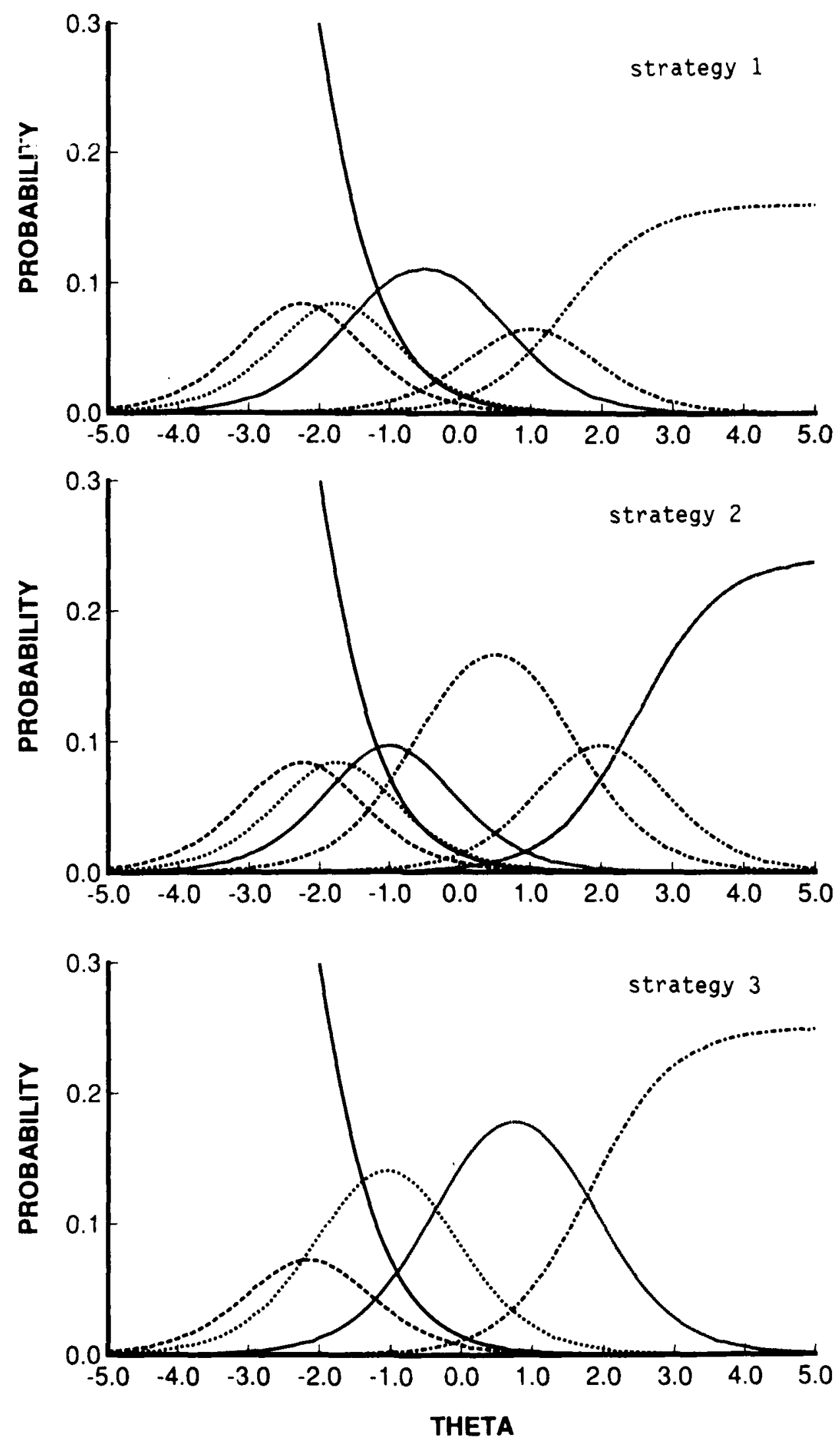
46
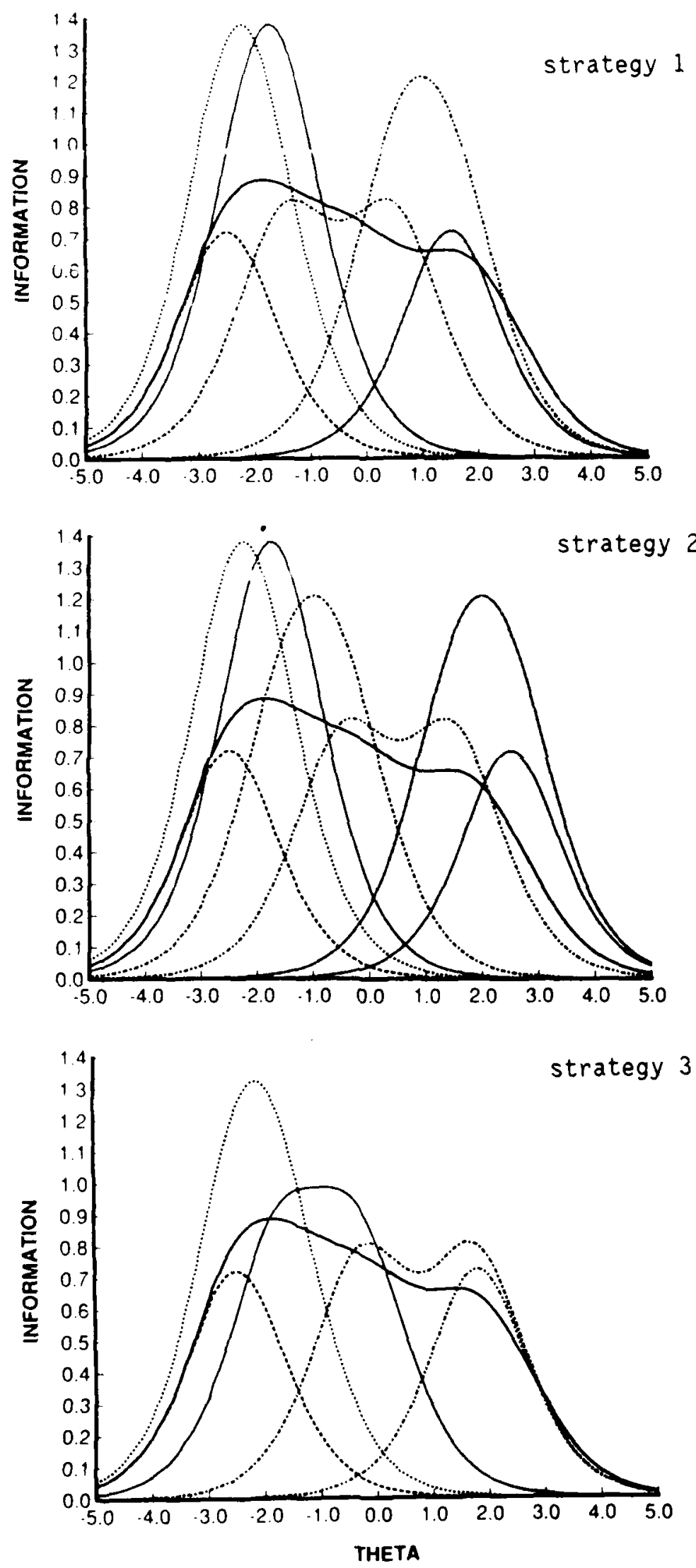
47
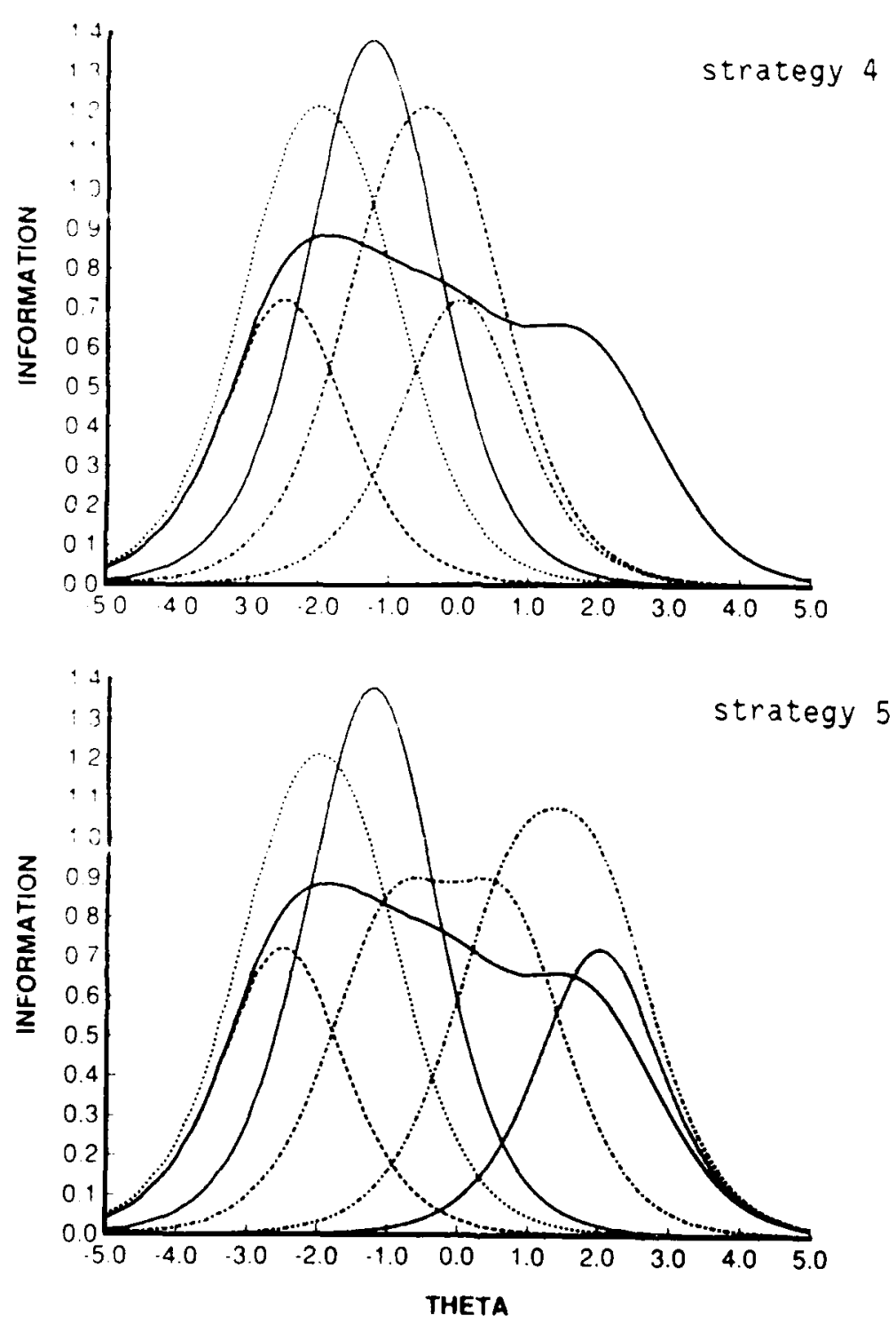

FIGURE $4-17$

Information Functions of the Subprocesses in Each of the Five Successful Strategies of the Example Whose Differential Strategy Tree Is Shown as Figure 1-2, Following the Logistic Model. 


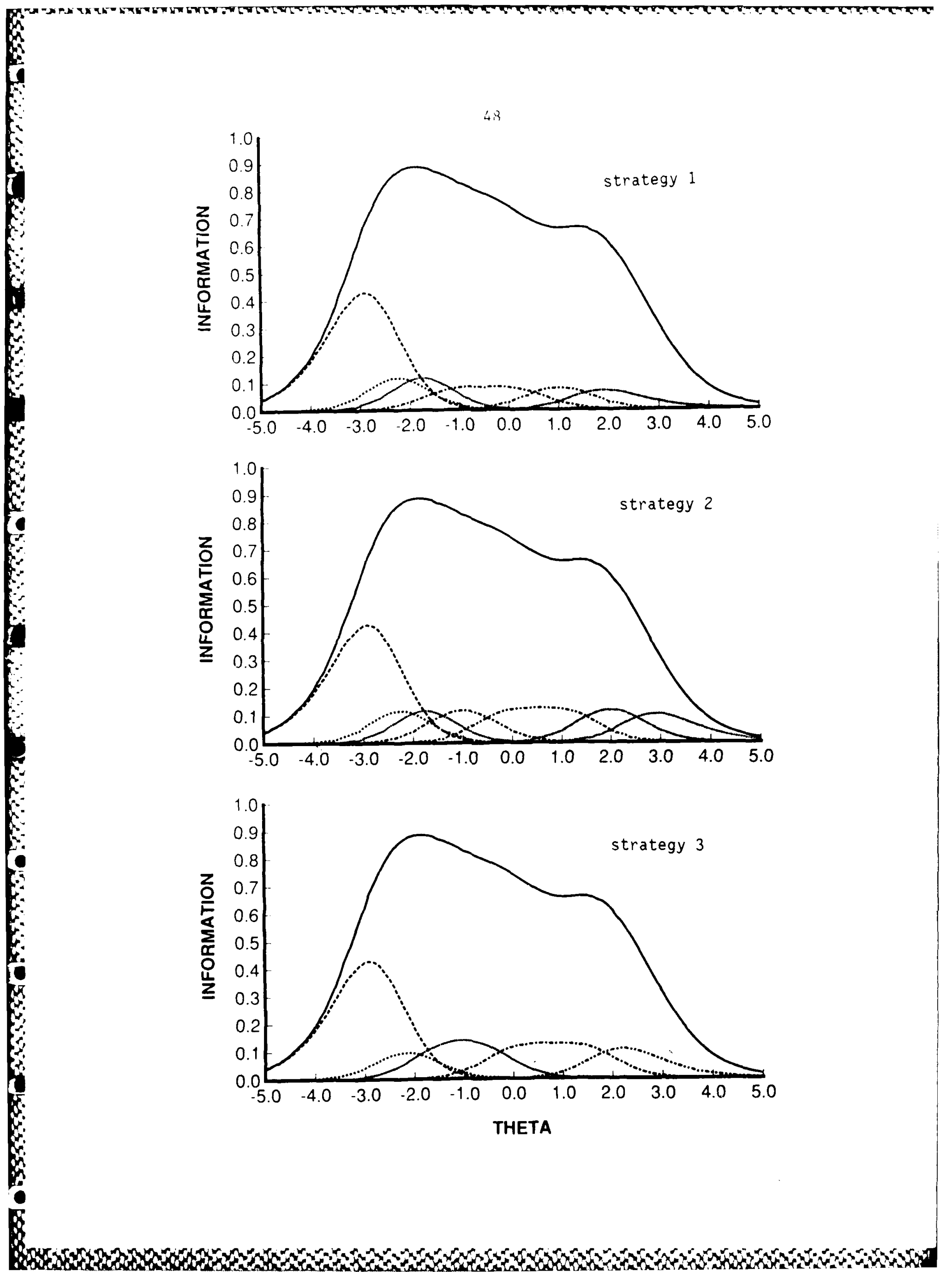




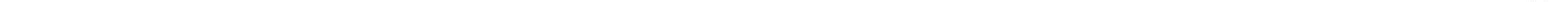




\section{Discussion}

A question may arise as to which estimate of the latent trait should be taken if the subject faltered from one strategy to another and did not reach the solution of the problem. One answer to this question may be to take the attainment score of the strategy that he took last, and use its corresponding operating characteristic in estimating his latent trait. Another answer may be to compare the resultant estimates of $\theta$ obtained by the separate strategies the subject has taken and select the highest estimate.

The usefulness of the proposed model is yet to discover. We need the collaboration of cognitive psychologists who are willing to collect data on larger samples, taking advantage of modern technologies. 


\section{REFERENCES}

[1] Samejima, F. A general model for the operating characteristic of graded item response. Chapel Hil1, North Carolina: The L. L. Thurstone Psychometric Laboratory Report, 55, 1967.

[2] Samejima, F. Estimation of latent ability using a response pattern of graded scores. Psychometrika Monograph, No. 17, 1969.

[3] Samejima, F. A generál model for free-response date. Psychometrika monograph, No. 18, 1972.

[4] Samejima F. Homogeneous case of the continuous response level. Psychometrika, 1973, 38, 203-219.

[5] Samejima, F. A general model for the homogeneous case of the continuous response. Office of Naval Research Report 83-3, 1983. 

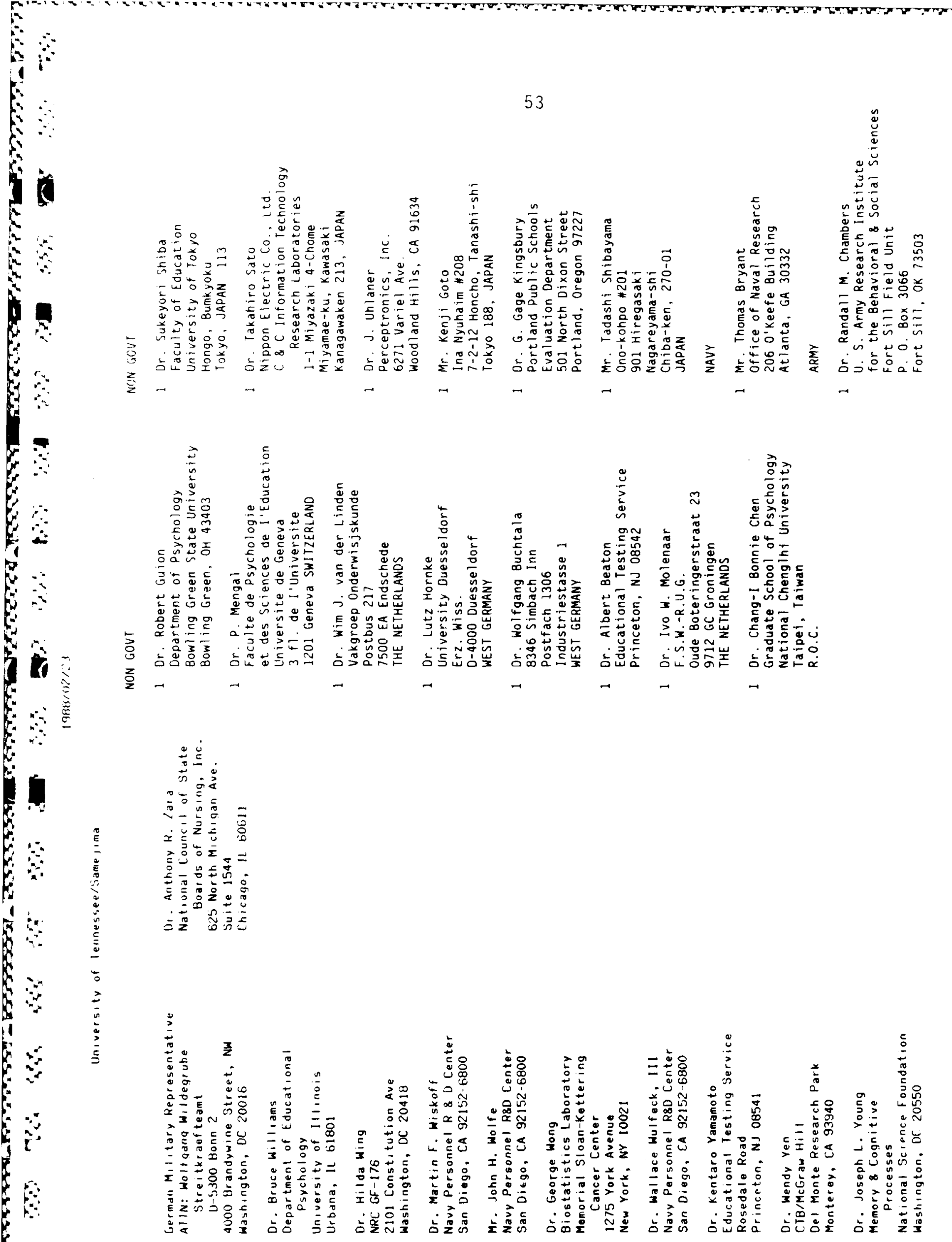

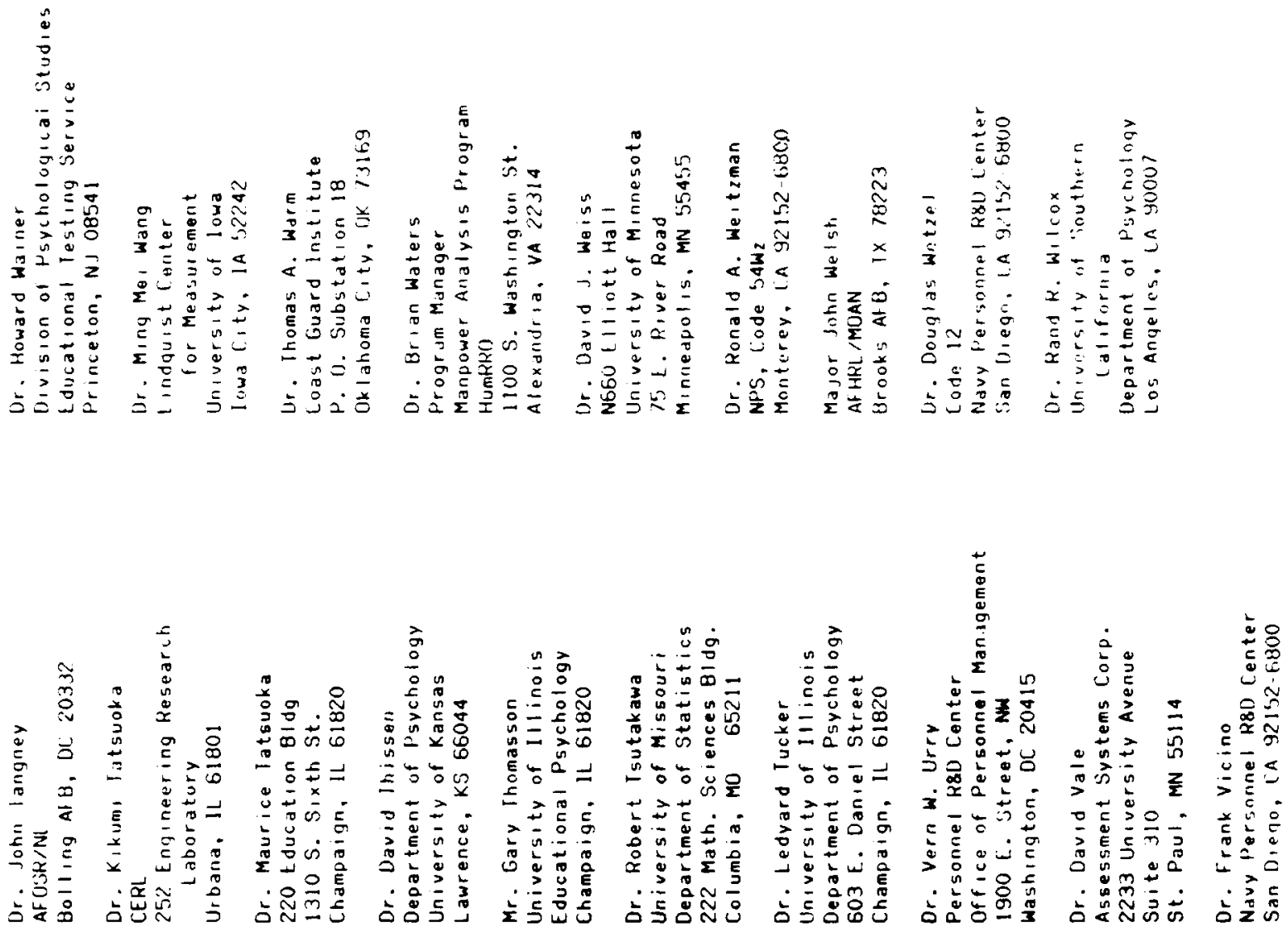

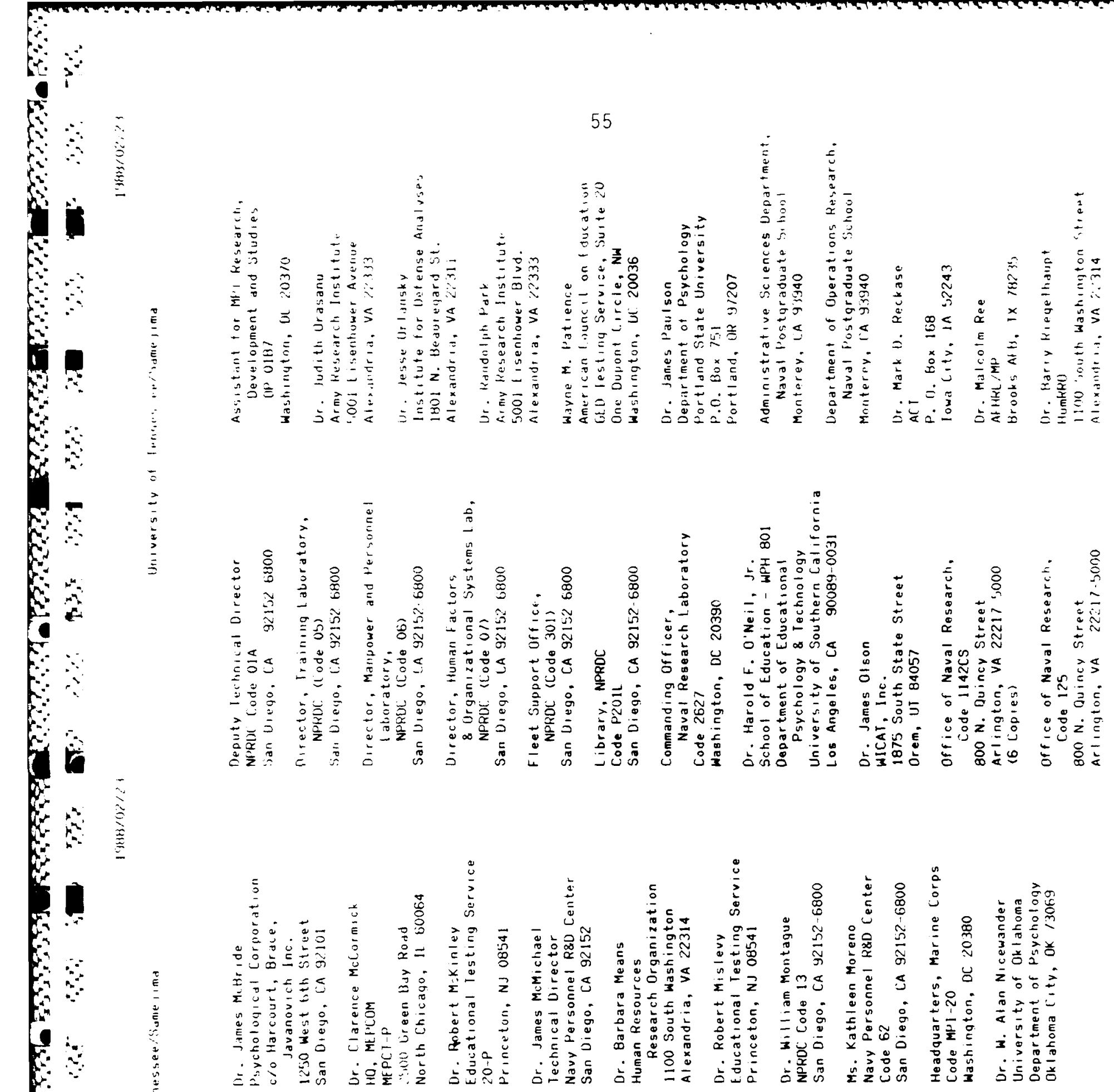

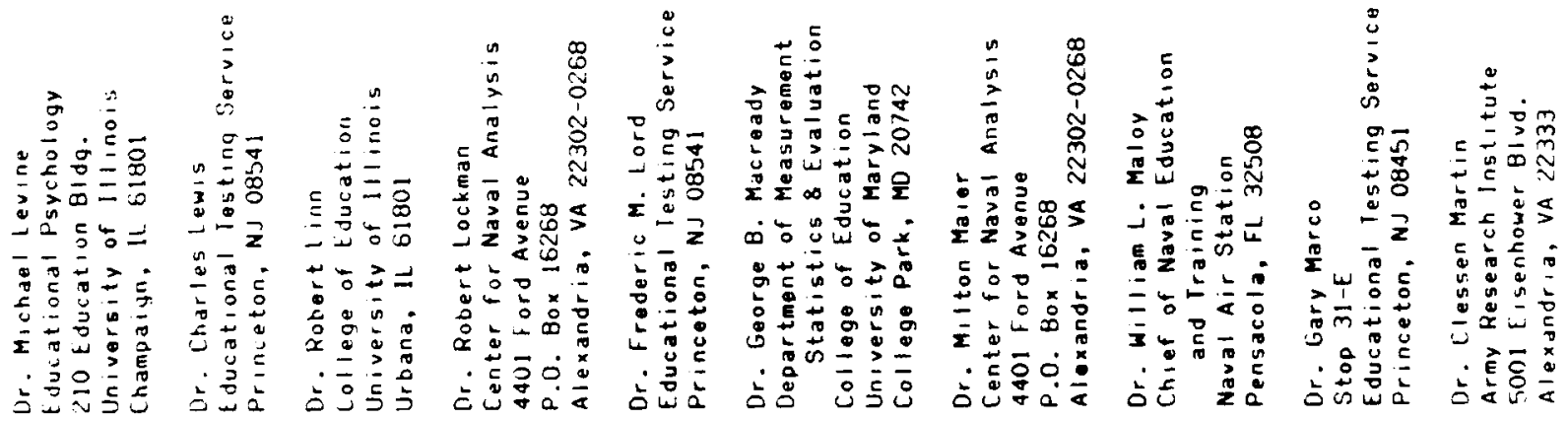




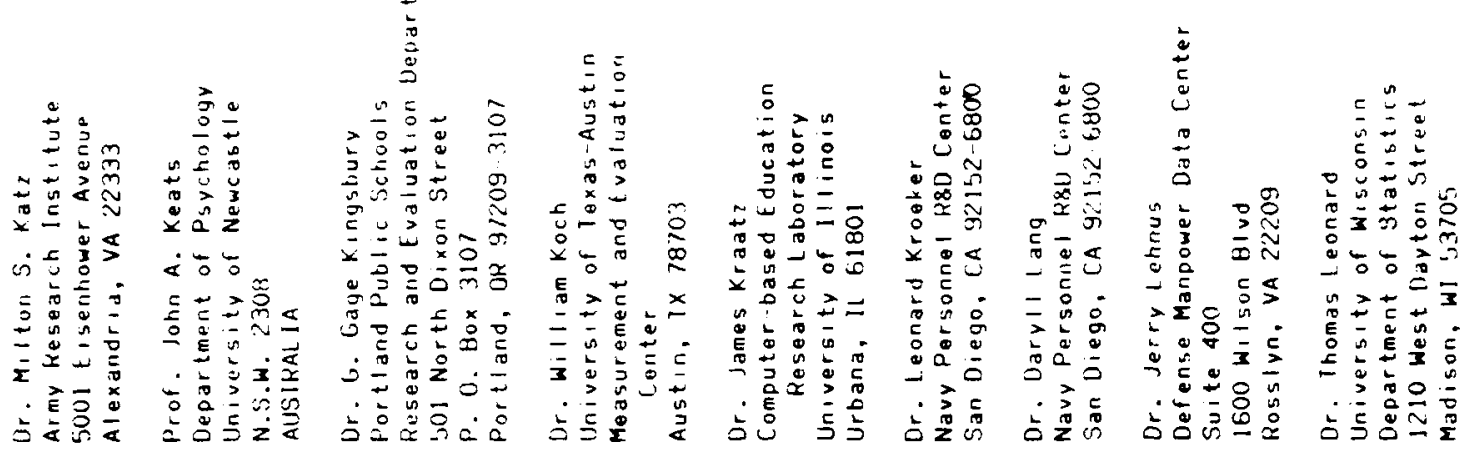

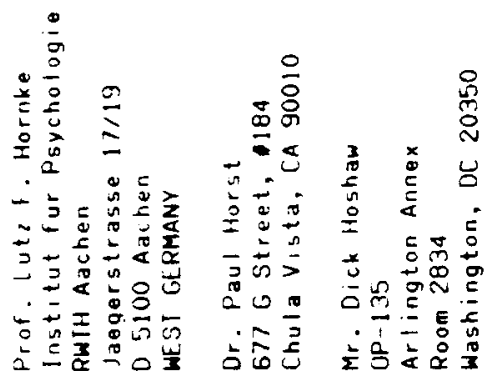

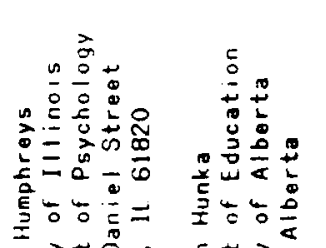

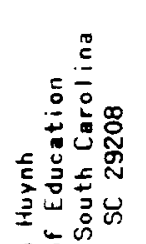
ำ

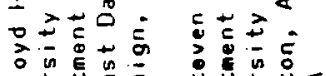

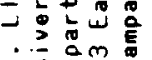

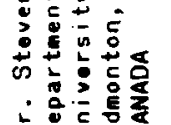

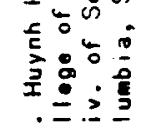

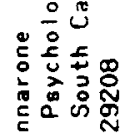

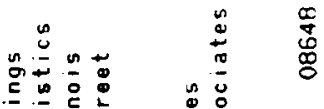

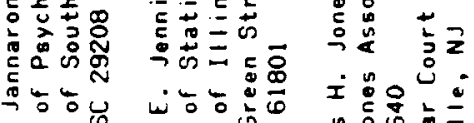

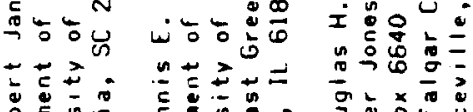

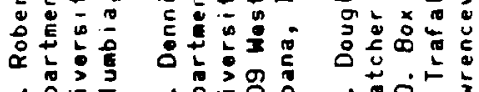

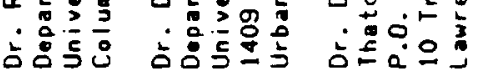

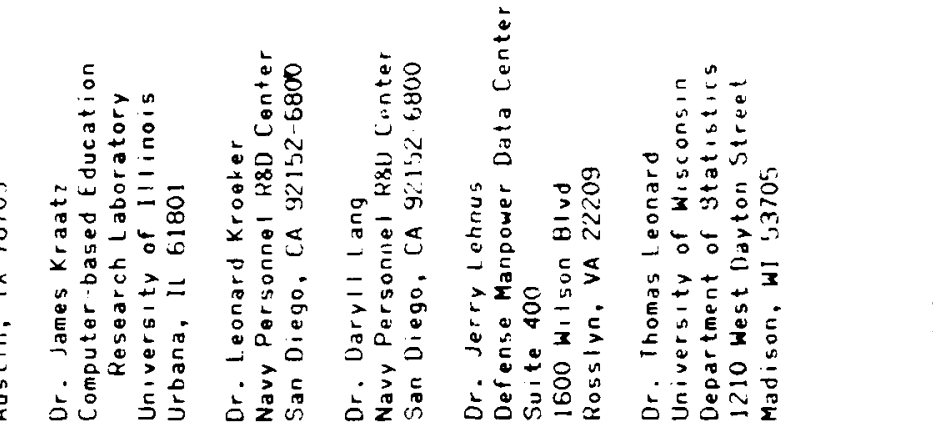

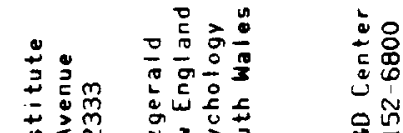




$$
\begin{aligned}
& \text { EN D } \\
& \text { DAEED } \\
& \text { FILM } \\
& 8-88 \\
& \text { DHIC }
\end{aligned}
$$

